\title{
Relations between tautological cycles on Jacobians
}

\author{
Ben Moonen
}

\begin{abstract}
We study tautological cycle classes on the Jacobian of a curve. We prove a new result about the ring of tautological classes on a general curve that allows, among other things, easy dimension calculations and leads to some general results about the structure of this ring. Further we lift a result of Herbaut and van der Geer-Kouvidakis to the Chow ring (as opposed to its quotient modulo algebraic equivalence) and we give a method to obtain further explicit cycle relations. As an ingredient for this we prove a theorem about how Polishchuk's operator $\mathscr{D}$ lifts to the tautological subalgebra of $\mathrm{CH}(J)$.
\end{abstract}

Mathematics Subject Classification (2000). 14C25, $14 \mathrm{H} 40$.

Keywords. Jacobian varieties, Chow ring, tautological cycles.

\section{Introduction}

Let $(J, \theta)$ be the Jacobian of a curve $C$ of genus $g \geq 2$, and let $j: C \hookrightarrow J$ be the embedding obtained by choosing a point $x_{0} \in C$. The Chow ring $\mathrm{CH}(J)$ (with $\mathbb{Q}$-coefficients) comes equipped with a number of structures: in addition to the usual intersection product we have the Pontryagin product $*$, the Fourier transform $\mathcal{F}$, and the action of $n^{*}$ and $n_{*}$ for all $n \in \mathbb{Z}$. Using the latter we obtain Beauville's decomposition $\mathrm{CH}(J)=\oplus \mathrm{CH}_{(j)}^{i}(J)$. These structures are inherited by the quotient $A(J):=\mathrm{CH}(J) / \sim_{\text {alg }}$.

In [2], Beauville studied the tautological subring $\mathcal{T}(C) \subset A(J)$, that can be defined as the smallest subalgebra that is stable under all operations just mentioned, and that contains the image of $A(C)$ under $j_{*}$. He proved that $\mathcal{T}(C)$ is generated by certain classes $p_{m}$ that are the components of $\mathcal{F}[C]$ in the Beauville decomposition. This leads to the question what is the ideal of relations between these classes.

The work of Polishchuk [15] provides us with a powerful method to produce relations. He considers the map $\phi: R \rightarrow \mathcal{T}(C)$ from the polynomial ring $R:=$ $\mathbb{Q}\left[x_{1}, x_{2}, \ldots\right]$ given by $x_{i} \mapsto p_{i}$. The elements of $R$ that are of degree $\geq g$ (with $x_{i}$ of degree $i$ ) with zero coefficient in front of $x_{1}^{g}$ lie in the kernel of $\phi$ for obvious reasons. But now the point is that Polishchuk is able to give an explicit differential operator $\mathscr{D}$ on $R$ that preserves the kernel of $\phi$, and that the ideal of "trivial" relations 
is far from stable under $\mathscr{D}$. Polishchuk studies the smallest ideal $I \subset R$ that is $\mathscr{D}$ stable and that contains all trivial relations. Conjecturally, for general curves $C$ (over a base field of sufficiently high transcendence degree) the quotient ring $\mathcal{R}:=R / I$ maps isomorphically to the tautological $\operatorname{ring} \mathcal{T}(C)$.

The main results of the present paper are the following:

First we give some new results on the structure of the ring $\mathcal{R}$. Though in principle it is not difficult to calculate, for a given $g$, the dimensions of all summands $\mathcal{R}_{(j)}^{i}$ on a computer, it is not so easy to obtain general conclusions about the structure of $\mathcal{R}$ from Polishchuk's methods. (In some sense the main obstacle is that the variable $x_{1}$ plays an exceptional role in the calculations.)

We prove a theorem, Theorem 2.6, that gives more insight in the structure of $\mathcal{R}$. Each $\mathcal{R}_{(j)}:=\bigoplus_{i} \mathcal{R}_{(j)}^{i}$ is a finite-dimensional $\mathfrak{s l}_{2}$-module. We give a simple recipe for the multiplicity of a given irreducible $\mathfrak{s l}_{2}$-module in each $\mathcal{R}_{(j)}$. As corollaries we get some general results about the relations in $\mathcal{R}_{(j)}$, especially for small and large (relative to $g$ ) values of $j$. Further we present a conjecture of van der Geer and Kouvidakis that gives a simple recipe for the dimensions of the spaces $\mathcal{R}_{(j)}^{i}$.

The second main goal of the paper is to lift some results about tautological cycle classes in $A(J)$ to the Chow ring of $J$. For general curves Polishchuk already obtained some results about this in [16]. He considered a subalgebra $\operatorname{taut}(C) \subset \mathrm{CH}(J)$ that we call the small tautological ring, and he proved that is generated by classes $p_{m}$ (lifting those considered above) and $q_{m}$ (essentially the Beauville components of $\mathscr{F}\left(j_{*} K\right)$, with $K$ the canonical class on $C$ ).

We get finer results if we make assumptions on the existence of a (special) $g_{d}^{r}$ on the curve. Herbaut [10] proved that in this case we get new relations between the classes $p_{m}$ modulo algebraic equivalence. This result was reproved and simplified by van der Geer and Kouvidakis in [9]. In itself, it is not very hard to lift the result of Herbaut and van der Geer-Kouvidakis to the Chow level. We do this in Section 4, following the method of [9] which is based on a Grothendieck-RiemannRoch calculation. The relation we obtain (see Theorem 4.6) is the following.

Theorem. Define $p_{n} \in \mathrm{CH}^{n}(J)$ as the degree $n$ component of $\mathcal{F}[j(C)]$, and for integers $i$ and $s$ define

$$
B(i, s)=\sum_{\substack{m_{1}, \ldots, m_{s} \\ m_{1}+\cdots+m_{s}=i}} m_{1} ! \ldots m_{s} ! \cdot p_{m_{1}} \ldots p_{m_{s}} .
$$

If $C$ has a $g_{d}^{r}$ then for all $i>d-r$ we have the relation

$$
B(i, r)=\sum_{n=1}^{i-1}(-1)^{n-1} c_{n}(\Gamma) \cdot B(i-n, r),
$$

where the $c_{n}(\Gamma) \in \mathrm{CH}^{n}(J)$ are certain classes (see (4.3)) associated to the $g_{d}^{r}$. 
The classes $c_{n}(\Gamma)$ in this result do not, in general, lie in the small tautological ring. So we are led to consider a bigger subalgebra Taut $(C) \subset \mathrm{CH}(J)$ that we call the big tautological ring. By definition it is the smallest $\mathbb{Q}$-subalgebra of $\mathrm{CH}(J)$ that is stable under all operations $\cdot, *, \mathcal{F}, n^{*}$ and $n_{*}$ and that contains the image of $j_{*}: \mathrm{CH}(C) \rightarrow \mathrm{CH}(J)$.

From the relations obtained in Theorem 4.6 we should like to obtain further ones by (repeated) application of an operator $\mathscr{D}$, lifting Polishchuk's operator on $A(J)$. In Section 3 we study the big tautological ring, and we define an operator $\mathscr{D}$ on it. As Taut $(C)$ is not finitely generated, it is now less easy to describe this operator explicitly. But the calculations we should like to perform involve only finitely many classes of the form $\mathcal{F}\left(j_{*} D_{i}\right)$ with $D_{i}$ divisors on $C$. We show that the subalgebra of Taut $(C)$ generated by these classes together with the $p_{m}$ and $q_{m}$ is stable under $\mathscr{D}$ and that $\mathscr{D}$ again acts on it as a differential operator that we can give explicitly.

The situation we arrive at is that we have lifted Herbaut's relations to the Chow level, and that we also have an explicit operator $\mathscr{D}$. Combined this gives an abundance of relations that can be calculated directly. We give some examples of the relations thus obtained in the final section. What remains is to get some control over the total ideal of relations thus obtained.

We would like to draw the reader's attention to some recent related work. In particular we would like to mention Polishchuk's paper [17], in which some ideas appear that are closely related to our Section 3, and the paper [8] of Fu and Herbaut, which contains some results related to the material in Section 4.

Acknowledgements. I thank Tom Koornwinder for helping me with Mathematica. I thank Gerard van der Geer and Alexis Kouvidakis for allowing me to present their conjecture on the dimensions of the spaces $\mathcal{R}_{(j)}^{i}$, see 2.13, and for explaining to me some details pertaining to their conjecture. Further I thank the anonymous referee for a number of corrections and suggestions that have led to some simplifications and to improvements in the exposition.

Notation and conventions. We work over a fixed algebraically closed field $k$. Throughout, if $X$ is a non-singular complete variety then by $\mathrm{CH}(X)$ we denote the Chow ring of $X$ tensored with $\mathbb{Q}$.

We write $\mathfrak{s l}_{2}=\mathbb{Q} \cdot f+\mathbb{Q} \cdot h+\mathbb{Q} \cdot e$ with $[e, f]=h,[e, h]=-2 e$, and $[f, h]=2 f$.

\section{The Chow ring of a Jacobian}

1.1. Let $C$ be a non-singular complete curve of genus $g \geq 2$. Let $J:=\mathrm{Pic}_{C / k}^{0}$ be its Jacobian, $\theta: J \stackrel{\sim}{\longrightarrow} J^{t}$ the canonical polarization. We also write $\theta \in \mathrm{CH}^{1}(J)$ for the 
class of a symmetric theta divisor. Choose a base point $x_{0} \in C$, and let $j: C \hookrightarrow J$ be the embedding given on points by $x \mapsto\left[O_{C}\left(x-x_{0}\right)\right]$.

Let $\mathcal{P}_{J}$ be the Poincaré bundle on $J \times J^{t}$. Let $\mathscr{L}_{J}$ be the line bundle on $J \times J$ obtained by pulling back $\mathcal{P}_{J}^{-1}$ under the map id ${ }_{J} \times \theta$. We have a Fourier transform $\mathscr{F}: \mathrm{CH}(J) \rightarrow \mathrm{CH}(J)$ given by $\mathscr{F}(x)=\operatorname{pr}_{2, *}\left(\operatorname{ch}\left(\mathscr{L}_{J}\right) \cdot \operatorname{pr}_{1}^{*}(x)\right)$. Further we have Beauville's decomposition $\mathrm{CH}^{i}(J)=\bigoplus_{j} \mathrm{CH}_{(j)}^{i}(J)$, with $x \in \mathrm{CH}_{(j)}^{i}(J)$ if and only if $n^{*}(x)=n^{2 i-j} \cdot x$ for all $n \in \mathbb{Z}$. The Fourier transform $\mathcal{F}$ induces a bijection between $\mathrm{CH}_{(j)}^{i}(J)$ and $\mathrm{CH}_{(j)}^{g-i+j}(J)$.

We write $[j(C)]$, or if there is no risk of confusion simply $[C]$, for the class of $j(C)$ in $\mathrm{CH}^{g-1}(J)$. Further, $[j(C)]_{(n)}$, or simply $[C]_{(n)}$, denotes the component of $[C]$ in $\mathrm{CH}_{(n)}^{g-1}(J)$.

1.2. We find it helpful to draw pictures representing the Chow ring as in Figure 1. The boxes $(i, j)$ represent the spaces $\mathrm{CH}_{(j)}^{i}$. In the horizontal direction we have the weight, where by definition $\mathrm{CH}_{(j)}^{i}$ has weight $2 i-j$. The Fourier transform acts as reflection in the central vertical line.

The filtration Fil ${ }^{\bullet}$ on $\mathrm{CH}(J)$ defined by $\mathrm{Fil}^{r} \mathrm{CH}:=\bigoplus_{j \geq r} \mathrm{CH}_{(j)}^{\bullet}$ should satisfy the conjectures of Beilinson and Murre; see Jannsen [12]. Most of the expected properties of this filtration are as yet unproved; we shall mention some of these.

It is known that $\mathrm{CH}_{(j)}^{i}$ can be nonzero only for $g-i \leq j \leq i$. According to a conjecture of Beauville in [1], §5, the spaces $\mathrm{CH}_{(j)}^{i}$ should be zero if $j<0$; this corresponds to one of the properties of Beilinson's conjectural filtration. In general this is known only for $i \in\{0,1, g-2, g-1, g\}$. Over finite fields or the algebraic closure of a finite field Beauville's conjecture is known; see Künnemann [13], Sections 7 and 8.

As $n^{*}$ acts on $H^{2 i}$ as multiplication by $n^{2 i}$, all classes in Fil ${ }^{1} \mathrm{CH}$ are homologically trivial. It should be the case that $\bigoplus_{i} \mathrm{CH}_{(0)}^{i}$ injects into cohomology, but this is not known in general. Similarly, by considering the weight we see that all classes in Fil $^{2} \mathrm{CH}$ map to zero under the Abel-Jacobi map, and it should be the case that $\mathrm{Fil}^{2} \mathrm{CH}$ is precisely the kernel of the Abel-Jacobi map.

The classes in $\mathrm{Fil}^{1} \mathrm{CH}^{g}$ represent 0-cycles of degree zero and are therefore algebraically trivial. By Fourier duality it follows that also all classes in $\mathrm{CH}_{(j)}^{j}$ with $j>0$ are algebraically trivial. In the picture these summands are indicated by boxes with a heavier border. As we shall see, in general there are many more classes that are algebraically trivial.

1.3. By the work of Künnemann in [13], the Chow motive of $J$ has a Lefschetz decomposition. This gives rise to an action of $\mathfrak{s l}_{2}$ on $\mathrm{CH}(J)$. We normalise this as 


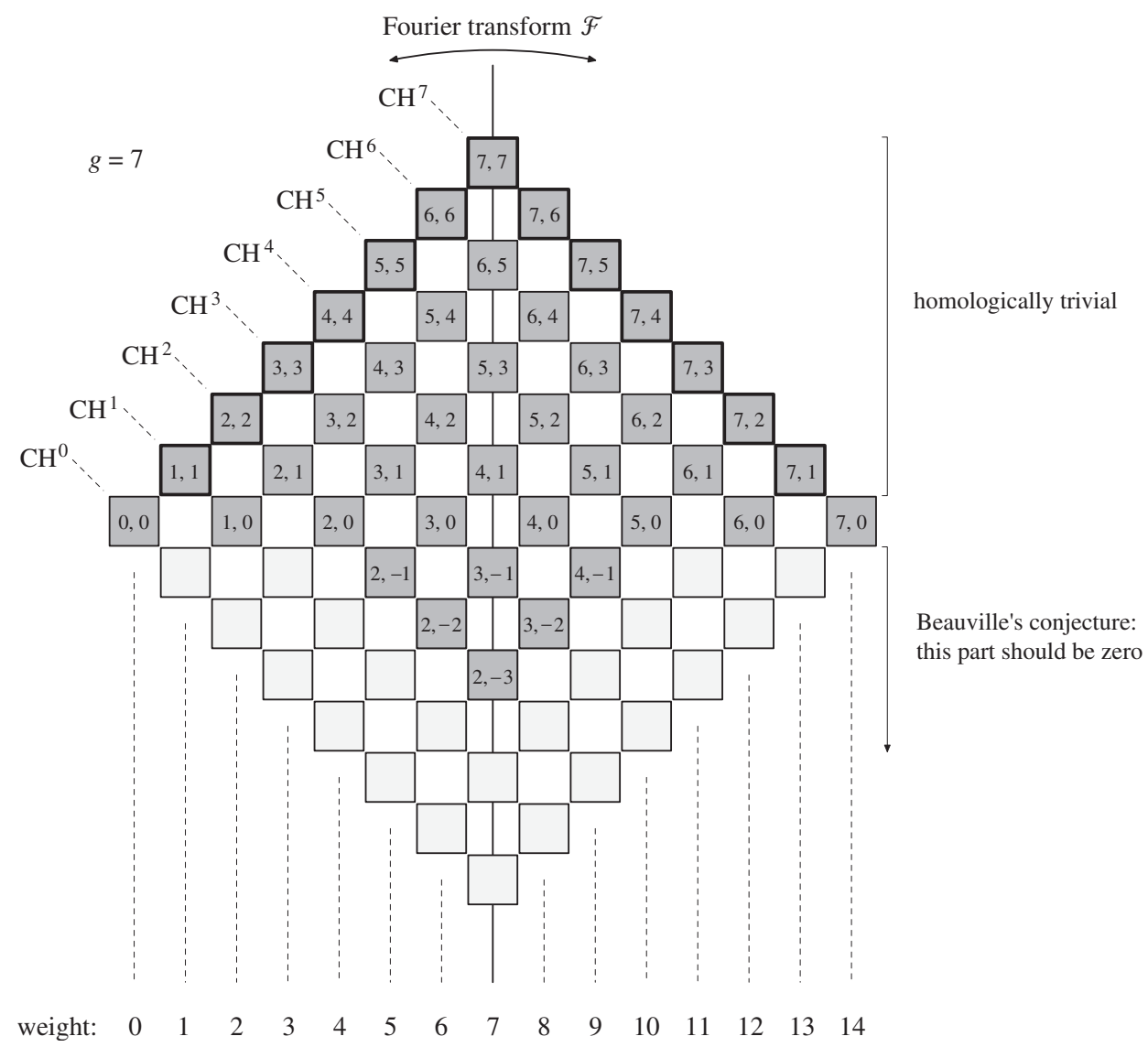

Figure 1. A picture of $\mathrm{CH}(J)$.

in [16]; so with notation given on p. 473 we have

$$
e(\alpha)=-\theta \cdot \alpha, \quad f(\alpha)=-[C]_{(0)} * \alpha,
$$

and

$$
h(\alpha)=(2 i-j-g) \cdot \alpha \quad \text { for } \alpha \in \mathrm{CH}_{(j)}^{i}(J) .
$$

1.4. Define $A(J)$ as the quotient of $\mathrm{CH}(J)$ modulo algebraic equivalence. This ring inherits all the structures on $\mathrm{CH}(J)$ that are relevant for us. Concretely, $A(J)$ has an intersection product, a Pontryagin product, a Fourier transform, a Beauville decomposition $A(J)=\oplus A_{(j)}^{i}$, and an $\mathfrak{s I}_{2}$-action. We shall use these structures without further comments. We again write $\theta$ for the class of a symmetric theta divisor in $A_{(0)}^{1}(J)$. 


\section{Cycle relations modulo algebraic equivalence - general curves}

2.1. Throughout this section, $g$ will be a fixed integer with $g \geq 2$. We start by reviewing some results from Polishchuk's paper [15].

Consider the polynomial $\operatorname{ring} R:=\mathbb{Q}\left[x_{1}, x_{2}, \ldots\right]$ in infinitely many variables. There are several gradings that will play a role in what follows. Among these are gradings that we call "codimension", "level" and "weight"; they are defined by setting

$$
\operatorname{codim}\left(x_{i}\right):=i, \quad \ell(\alpha):=i-1, \quad \text { and } \quad w\left(x_{i}\right):=i+1 .
$$

We have $w=2 \cdot \operatorname{codim}-\ell$.

Define $R_{(j)}^{i}:=\{\alpha \in R \mid \operatorname{codim}(\alpha)=i$ and $\ell(\alpha)=j\}$. This gives a bigrading $R=\oplus R_{(j)}^{i}$. We write $R^{i}:=\bigoplus_{j} R_{(j)}^{i}$ and $R_{(j)}:=\bigoplus_{i} R_{(j)}^{i}$. Let Fil ${ }^{\bullet}$ be the descending filtration by level, so Fil ${ }^{n} R:=\bigoplus_{j \geq n} R_{(j)}$.

Define an operator $\mathscr{D}$ on $R$ by

$$
\mathscr{D}:=-g \partial_{1}+\frac{1}{2} \sum_{m, n \geq 1}\left(\begin{array}{c}
m+n \\
n
\end{array}\right) x_{m+n-1} \partial_{m} \partial_{n},
$$

where $\partial_{k}:=\partial_{x_{k}}$. We give $R$ the structure of an $\mathfrak{s l}_{2}$-module by setting

$$
\begin{aligned}
& e(\alpha)=x_{1} \cdot \alpha \\
& h(\alpha)=(w(\alpha)-g) \cdot \alpha=(2 i-j-g) \cdot \alpha \quad \text { if } \alpha \in R_{(j)}^{i} ; \\
& f(\alpha)=-\mathscr{D}(\alpha) .
\end{aligned}
$$

See [15], Lemma 3.2, and see p. 473 for our notation regarding $\mathfrak{s} \mathfrak{l}_{2}$.

Define $I \subset R$ to be the smallest linear subspace that is stable under $\mathscr{D}$ and that contains $R^{>g}+$ Fil $^{1} R^{g}$. Concretely, $I=R^{>g} \oplus \sum_{n \geq 0} \mathscr{D}^{n}\left(\mathrm{Fil}^{1} R^{g}\right)$. Polishchuk shows that $I=\cap_{n \geq 0} \operatorname{Im}\left(\mathscr{D}^{n}\right)$, and that $I$ is in fact an ideal of $R$. Define $\mathcal{R}:=R / I$. (This is the ring called $R_{g}^{\mathrm{Jac}}$ in [15], but for later use it will be convenient to have a simpler notation.) The ring $\mathcal{R}$ inherits a bigrading $\mathcal{R}=\oplus \mathcal{R}_{(j)}^{i}$ and the structure of an $\mathfrak{s l}_{2}$-module. Note that the subspaces $\mathcal{R}_{(j)}$ are $\mathfrak{s l}_{2}$-submodules of $\mathcal{R}$.

The next ingredient is that we have a "Fourier operator" $\mathcal{F}$ on $\mathcal{R}$, given by [15], formula (0.4). (Our $\mathcal{F}$ is Polishchuk's $S$.) If $\alpha \in \mathcal{R}_{(j)}^{i}$ then $\mathcal{F}^{2}(\alpha)=(-1)^{g+j} \alpha$.

2.2. Let now $C$ be a non-singular curve of genus $g$. We use the notation of 1.1. Consider the homomorphism of $\mathbb{Q}$-algebras $\phi: R \rightarrow A(J)$ given by $\phi\left(x_{i}\right)=p_{i}$, with

$$
p_{i}:=\text { degree } i \text { component of } \mathcal{F}[C]=\mathcal{F}\left([C]_{(i-1)}\right) .
$$

The image of $\phi$ is the tautological subring introduced and studied by Beauville in [2]. We shall denote this tautological ring by $\mathcal{T}(C):=\operatorname{Im}(\phi) \subset A(J)$. 
We have $I \subset \operatorname{Ker}(\phi)$. Let $\psi: \mathcal{R} \rightarrow A(J)$ be the induced homomorphism. This map $\psi$ is compatible with all structures considered above; that is,

- $\psi\left(\mathcal{R}_{(j)}^{i}\right) \subseteq A_{(j)}^{i}(J)$ for all $i, j$;

- $\psi$ is $\mathfrak{s l}_{2}$-equivariant;

$-\psi \circ \mathscr{F}=\mathscr{F} \circ \psi$.

2.3. The tautological ring $\mathcal{T}(C) \subset A(J)$ is generated by the classes $p_{1}, \ldots, p_{g}$. We should like to understand the relations between these classes. The relations in Polishchuk's ideal $I$ are those that are obtained from "trivial relations", i.e., relations $F\left(p_{1}, p_{2}, \ldots\right)=0$ with $F \in R^{>g}+\mathrm{Fil}^{1} R^{g}$, by (repeated) application of the operator $\mathscr{D}$. The relations thus obtained are in many cases non-trivial from a geometric perspective. For instance, using this method Polishchuk shows that $p_{n}=0$ in $A(J)$ for all $n \geq \frac{g}{2}+1$. (These particular relations also follow from a theorem of Colombo and van Geemen in [4], as the gonality of $C$ is at most $1+\lceil g / 2\rceil$.) Polishchuk conjectures that for a generic curve $C$ (over an algebraically closed base field of sufficiently high transcendence degree over the prime field) the ideal $I$ gives all relations.

For a given $g$ we can, at least in principle, write down a basis for the ideal $I$ over $\mathbb{Q}$. See [15], Section 2.6 for examples in genera $\leq 10$. The drawback of this method is that it is purely computational, and that it is hard to get an insight in the structure of $\mathcal{R}$. For instance, in examples one finds that there are many pairs $(i, j)$, especially $j \geq g / 2$, for which $\mathcal{R}_{(j)}^{i}=0$, and one would like to understand precisely for which pairs this happens.

Our main result in this section further details Polishchuk's method, and gives the structure of the ring $\mathcal{R}$ as an $\mathfrak{s l}_{2}$-module. Using our theorem, it becomes very easy to calculate the dimensions of the $\mathcal{R}_{(j)}^{i}$, and we also get some general results about the structure of $\mathcal{R}$.

We start with a simple lemma.

2.4 Lemma. Let $\alpha \in R_{(j)}^{i}$. Then

$$
\begin{aligned}
& \mathscr{D}^{n}\left(x_{1}^{a} \cdot \alpha\right) \\
& \quad=\sum_{s=0}^{\min (n, a)} \frac{n !}{(n-s) !} \frac{a !}{(a-s) !} \cdot\left(\begin{array}{c}
2 i-j-g+a-n+s-1 \\
s
\end{array}\right) \cdot x_{1}^{a-s} \mathbb{D}^{n-s}(\alpha) .
\end{aligned}
$$

for all $n \geq 0$ and $a \geq 0$.

Note that the binomial coefficient has to be taken in the generalised sense, as $2 i-j-g+a-n+s-1$ may be negative. As a particular case of the lemma, we have

$$
\mathscr{D}\left(x_{1}^{a} \cdot \alpha\right)=x_{1}^{a} \cdot \mathscr{D}(\alpha)+a(2 i-j-g+a-1) \cdot x_{1}^{a-1} \alpha .
$$


Proof. The formula is proven by an easy induction on $n$. To start the induction we first prove (1) by induction on $a$, using that $\mathscr{D}\left(x_{1} \beta\right)-x_{1} \mathscr{D}(\beta)=(2 i-j-g) \cdot \beta$ for $\beta \in R_{(j)}^{i}$.

2.5. Given an integer $j \geq 0$, define $\operatorname{Mon}_{(j)} \subset R_{(j)}$ to be the (finite) set of monomials in the variables $x_{2}, x_{3}, \ldots$ (no $\left.x_{1}\right)$ with $\ell(\alpha)=j$. For $j=0$ we have $\operatorname{Mon}_{(j)}=\{1\}$. Let $\operatorname{Mon}_{(j)}^{i}:=\operatorname{Mon}_{(j)} \cap R_{(j)}^{i}$.

If $j \geq 1$ there is a bijection between $\operatorname{Mon}_{(j)}$ and the set of partitions $j=$ $j_{1}+j_{2}+\cdots+j_{r}$ with $1 \leq j_{1} \leq j_{2} \leq \cdots \leq j_{r}$, letting such a partition correspond to $\alpha=x_{j_{1}+1} x_{j_{2}+1} \ldots x_{j_{r}+1}$. Under this bijection, $\operatorname{Mon}_{(j)}^{i}$ corresponds with the partitions with $i-j$ parts. In particular, $\operatorname{Mon}_{(j)}^{i}$ is non-empty (still for $j \geq 1$ ) if and only if $j+1 \leq i \leq 2 j$.

The set $\operatorname{Mon}_{(j)}$ is a basis for $R_{(j)}$ as a $\mathbb{Q}\left[x_{1}\right]$-module. Write $M_{(j)}^{i}$ for the $\mathbb{Q}$-vector space with basis $\operatorname{Mon}_{(j)}^{i}$; similarly, let $M_{(j)}=\bigoplus_{i} M_{(j)}^{i}$ be the $\mathbb{Q}$-vector space generated by the set $\operatorname{Mon}_{(j)}$. Note that $\mathscr{D}\left(M_{(j)}^{i}\right) \subseteq M_{(j)}^{i-1}$.

2.6 Theorem. Write St for the tautological 2-dimensional representation of $\mathfrak{s l}_{2}$. Given $i$ and $j$ with $2 i-j \leq g$, write

$$
\mu(i, j):=\operatorname{dim}_{\mathbb{Q}}\left(M_{(j)}^{i} / D^{g-2 i+j+1}\left(M_{(j)}^{g-i+j+1}\right)\right) .
$$

Then for all $j \geq 0$ we have

$$
\mathcal{R}_{(j)} \cong \bigoplus_{i=j}^{\min \left(g-1,2 j,\left\lfloor\frac{g+j}{2}\right\rfloor\right)}\left[\mathrm{Sym}^{g-2 i+j}(\mathrm{St})\right]^{\mu(i, j)}
$$

as $\mathfrak{s} \mathfrak{l}_{2}$-modules.

Proof. The result for $j=0$ says that $\mathcal{R}_{(0)} \cong \operatorname{Sym}^{g}(\mathrm{St})$, which is immediate. In the rest of the proof we shall assume that $j \geq 1$. Let $\Phi$ • be the ascending filtration of $R_{(j)}$ by $\mathbb{Q}\left[x_{1}\right]$-submodules that is given by $\Phi_{i}:=\mathbb{Q}\left[x_{1}\right] \cdot\left(\bigoplus_{k \leq i} M_{(j)}^{k}\right)$. Note that the $\Phi_{i}$ are $\mathfrak{s I}_{2}$-submodules of $\mathcal{R}_{(j)}$, and that $\Phi_{j}=(0)$ and $\Phi_{2 j}=R_{(j)}$. Let $\Psi \bullet$ be the filtration on $\mathcal{R}_{(j)}$ induced by $\Phi$.

If $\alpha \in \operatorname{Mon}_{(j)}^{i}$ then $\mathscr{D}^{n}(\alpha) \in \Phi_{i-1}$ for all $n \geq 1$. Hence by Lemma 2.4 we have

$$
\mathscr{D}^{n}\left(x_{1}^{a} \cdot \alpha\right) \equiv \begin{cases}0 & \text { if } a<n \\
\frac{a !}{(a-n) !}\left(\begin{array}{c}
2 i-j-g+a-1 \\
n
\end{array}\right) x_{1}^{a-n} \alpha & \text { if } a \geq n\end{cases}
$$

modulo $\Phi_{i-1}$. Write $V_{\alpha}$ for the image of $\mathbb{Q}\left[x_{1}\right] \cdot \alpha$ in $\operatorname{gr}_{i}^{\Phi}$, which is an $\mathfrak{s l}_{2}$-submodule. We find: 
- If $2 i-j>g$ then $V_{\alpha}$ is irreducible, $\operatorname{dim}\left(V_{\alpha}\right)=\infty$.

- If $2 i-j \leq g$ then $V_{\alpha}$ is an extension,

$$
0 \longrightarrow U_{\alpha} \longrightarrow V_{\alpha} \longrightarrow W_{\alpha} \longrightarrow 0
$$

here $U_{\alpha}:=\operatorname{Im}\left(\mathbb{Q}\left[x_{1}\right] \cdot x_{1}^{g-2 i+j+1} \alpha\right)$ is infinite dimensional and irreducible, and $W_{\alpha}$ is isomorphic with $\operatorname{Sym}^{g-2 i+j}(\mathrm{St})$.

As $\mathcal{R}_{(j)}$ is finite dimensional it follows in particular that $\Phi_{\left\lfloor\frac{g+j}{2}\right\rfloor}$ surjects to $\mathcal{R}_{(j)}$.

Let $i$ be an integer with $j+1 \leq i \leq \min \left(2 j,\left\lfloor\frac{g+j}{2}\right\rfloor\right)$. The set $\operatorname{Mon}_{(j)}^{i}$ gives a basis for $\operatorname{gr}_{i}^{\Phi}$ as a $\mathbb{Q}\left[x_{1}\right]$-module, and by what we have just seen we have an exact sequence

$$
0 \longrightarrow U \longrightarrow \operatorname{gr}_{i}^{\Phi} \longrightarrow W \longrightarrow 0
$$

where $W$ is the direct sum of the spaces $W_{\alpha}$ for $\alpha \in \operatorname{Mon}_{(j)}^{i}$. Consider the subspace $M_{(j)}^{i} \subset \Phi_{i}$. Let $\bar{M}_{(j)}^{i}$ be the image of $M_{(j)}^{i}$ under the composition $\Phi_{i} \rightarrow \mathrm{gr}_{i}^{\Phi} \rightarrow W$. The natural map $M_{(j)}^{i} \rightarrow \bar{M}_{(j)}^{i}$ is an isomorphism. With $f$ as on p. 473 we have $\bar{M}_{(j)}^{i}=\operatorname{Ker}\left(f_{\mid W}\right)$, and $W \cong \bar{M}_{(j)}^{i} \otimes_{\mathbb{Q}} \operatorname{Sym}^{g-2 i+j}(\mathrm{St})$ as $\mathfrak{s l}_{2}$-modules.

Clearly the natural map $\operatorname{gr}_{i}^{\Phi} \rightarrow \operatorname{gr}_{i}^{\Psi}$ factors via a map $\xi: W \rightarrow \operatorname{gr}_{i}^{\Psi}$. Let $K:=\operatorname{Ker}(\xi)$, which is an $\mathfrak{s l}_{2}$-submodule of $W$. Let $K[f]:=\operatorname{Ker}\left(f_{\mid K}\right)$. Then we have $K[f]=\operatorname{Ker}(\xi) \cap \bar{M}_{(j)}^{i}$, and $\operatorname{gr}_{i}^{\Psi}$ is isomorphic with $\left(\bar{M}_{(j)}^{i} / K[f]\right) \otimes_{\mathbb{Q}}$ $\mathrm{Sym}^{g-2 i+j}(\mathrm{St})$ as $\mathfrak{s l}_{2}$-modules.

Now consider the composition

$$
M_{(j)}^{i} \stackrel{\sim}{\longrightarrow} \bar{M}_{(j)}^{i} \longleftrightarrow W \longrightarrow \operatorname{gr}_{i}^{\Psi},
$$

which is the just the restriction of the natural map $\Phi_{i} \rightarrow \mathrm{gr}_{i}^{\Psi}$ to $M_{(j)}^{i} \subset \Phi_{i}$. An element $y \in M_{(j)}^{i}$ maps to zero under this map if and only if $y \in I+\Phi_{i-1}$. So to complete the proof, it suffices to show that for $y \in M_{(j)}^{i}$ we have

$$
\begin{aligned}
y \in I+\Phi_{i-1} & \Longleftrightarrow y \in\left(I \cap R_{(j)}^{i}\right)+x_{1} R_{(j)}^{i-1} \\
& \Longleftrightarrow y \in D^{g-i}\left(R_{(j)}^{g}\right)+x_{1} R_{(j)}^{i-1} \\
& \Longleftrightarrow y \in\left(\sum_{a=0}^{i-j-1} \mathbb{D}^{g-i-a}\left(M_{(j)}^{g-a}\right)\right)+x_{1} R_{(j)}^{i-1} \\
& \Longleftrightarrow y \in D^{g-2 i+j+1}\left(M_{(j)}^{g-i+j+1}\right)+x_{1} R_{(j)}^{i-1} \\
& \Longleftrightarrow y \in D^{g-2 i+j+1}\left(M_{(j)}^{g-i+j+1}\right) .
\end{aligned}
$$

For (a), the implication " $\Leftarrow$ " is clear, as $x_{1} R_{(j)}^{i-1} \subseteq \Phi_{i-1}$ and $I \cap R_{(j)}^{i} \subseteq I$. For the converse, suppose we have $z \in I$ and $w \in \Phi_{i-1}$ with $y=z+w$. Because $I$ 
and $\Phi_{i-1}$ are bi-homogeneous with respect to the decomposition $R=\oplus R_{(j)}^{i}$, we may replace $z$ and $w$ by their components in $R_{(j)}^{i}$, in which case $z \in I \cap R_{(j)}^{i}$ and $w \in x_{1} \cdot R_{(j)}^{i-1}$.

For (b), just note that $I \cap R_{(j)}^{i}=\mathscr{D}^{g-i}\left(R_{(j)}^{g}\right)$. For (c), which is really the main point, start with the decomposition $R_{(j)}^{g}=\bigoplus_{a \geq 0} x_{1}^{a} \cdot M_{(j)}^{g-a}$. If $\alpha \in M_{(j)}^{g-a}$ then using Lemma 2.4 we find that

$$
\mathscr{D}^{g-i}\left(x_{1}^{a} \alpha\right)= \begin{cases}0 & \text { if } a \geq i-j \\
\frac{(g-i) ! a !}{(g-i-a) !}\left(\begin{array}{c}
i-j-1 \\
a
\end{array}\right) \mathscr{D}^{g-i-a}(\alpha) & \text { for } 0 \leq a \leq i-j-1,\end{cases}
$$

modulo $x_{1} \cdot R_{(j)}^{i-1}$.

Finally, (d) follows from the remark that $\mathscr{D}\left(M_{(j)}^{b}\right) \subseteq M_{(j)}^{b-1}$ for all $b$, and for (e) we use that $y$ and $\mathscr{D}^{g-2 i+j+1}\left(M_{(j)}^{g-i+j+1}\right)$ are both contained in $M_{(j)}^{i}$, whereas $M_{(j)}^{i} \cap x_{1} R_{(j)}^{i-1}=0$.

2.7 Remarks. (i) One of the main advantages of the result is that the calculations do not involve the variable $x_{1}$, and that therefore the operator $\mathscr{D}$ gets a much simpler form. We give some corollaries below. It should be possible to get even finer results; see 2.13 for some speculation. The only obstacle for pushing our results further is of a purely combinatorial nature.

(ii) The theorem should not be read as saying that the images of the monomial spaces $M_{(j)}^{i}$ in $\mathcal{R}_{(j)}^{i}$ consist of primitive classes, i.e., classes in the kernel of $f$. This is simply not the case. However, to an element $\alpha \in M_{(j)}^{i}$ corresponds a primitive class $\alpha^{\prime}$, given by

$$
\alpha^{\prime}=\sum_{n \geq 0} \frac{\left(-x_{1}\right)^{n} \cdot \mathscr{D}^{n}(\alpha)}{(n !)^{2} \cdot\left(\begin{array}{c}
2 i-j-g-2 \\
n
\end{array}\right)}
$$

(Note that the sum is finite, as $\mathscr{D}^{n}(\alpha)=0$ for $n \gg 0$.)

(iii) For $j \geq 1$ the direct sum in (2) starts at $i=j+1$. To "visualize" where the various summands $M_{(j)}^{i}$ and $R_{(j)}^{i}$ lie, for instance in the definition of the multiplicity $\mu(i, j)$, it is usually helpful to look at a picture, as in Figure 1. Recall that the number $2 i-j$ is the weight. The summand $R_{(j)}^{g-i+j}$ is the Fourier mirror image of $R_{(j)}^{i}$. The reader is encouraged to look at the examples in 2.12 below.

2.8 Corollary. Write $\mathcal{R}[g]$ for the ring $\mathcal{R}$ in genus $g$. If $2 i-j \leq g$ and $g<g^{\prime}$ then the multiplicity of $\mathrm{Sym}^{g^{\prime}-2 i+j}(\mathrm{St})$ in $\mathcal{R}\left[g^{\prime}\right]_{(j)}$ is greater or equal to the multiplicity of $\mathrm{Sym}^{g-2 i+j}(\mathrm{St})$ in $\mathcal{R}[g]_{(j)}$.

Proof. Note that

$$
\mathscr{D}^{g^{\prime}-2 i+j+1}: M_{(j)}^{g^{\prime}-i+j+1} \rightarrow M_{(j)}^{i}
$$


factors through

$$
\mathbb{D}^{g-2 i+j+1}: M_{(j)}^{g-i+j+1} \rightarrow M_{(j)}^{i}
$$

2.9 Corollary. For $n \leq \frac{g+1}{2}$ we have $x_{n} \neq 0$ in $\mathcal{R}$.

Proof. Recall that $x_{n} \in M_{(n-1)}^{n}$. In the proof of Theorem 2.6 we have seen that the kernel of the map $M_{(j)}^{i} \rightarrow \operatorname{gr}_{i}^{\Psi}$ equals $\mathscr{D}^{g-2 i+j+1}\left(M_{(j)}^{g-i+j+1}\right)$. For $i=n$ and $j=n-1$, this says that the kernel of the map $M_{(n-1)}^{n} \rightarrow \operatorname{gr}_{n}^{\Psi}$ equals $\mathscr{D}^{g-n}\left(M_{(n-1)}^{g}\right)$. But $M_{(n-1)}^{g}=0$ for $n \leq \frac{g+1}{2}$, so the image of $x_{n}$ in $\operatorname{gr}_{n}^{\Psi}$ (and a fortiori also the image in $\mathcal{R}$ ) is nonzero.

Of course it is far more interesting to have results about the non-vanishing of classes $p_{n}$ in $\mathrm{CH}(J)$, for a general curve $C$. Ceresa's theorem in [3] gives such a result, as it tells us that for $g \geq 3$ and general $C$ we have $p_{2} \neq 0$ in $A(J)$. (This follows from [3] using [10], Theorem 5.) See Fakhruddin [7] and Ikeda [11] for some further results.

2.10 Corollary. (i) If $i+j \leq g$ then the natural map $R_{(j)}^{i} \rightarrow \mathcal{R}_{(j)}^{i}$ is an isomorphism. have

(ii) If $0 \leq j \leq g / 3$ then $R_{(j)}^{i} \cong \mathcal{R}_{(j)}^{i}$ for all $i$ with $2 i-j \leq g$. In this case we

$$
\mathcal{R}_{(j)} \cong \bigoplus_{i=j+1}^{2 j}\left[\operatorname{Sym}^{g-2 i-j}(\mathrm{St})\right]^{v(i, j)}
$$

as $\mathfrak{s l}_{2}$-modules, where $v(i, j)$ is the number of ordered partitions of $j$ with $i-j$ parts.

Proof. By definition, for any pair $(i, j)$ the map $R_{(j)}^{i} \rightarrow \mathcal{R}_{(j)}^{i}$ is surjective, so it is an isomorphism if and only if the two spaces have the same dimension. Note that Fourier duality gives isomorphisms $R_{(j)}^{i} \stackrel{\sim}{\longrightarrow} R_{(j)}^{g-i+j}$ and $\mathcal{R}_{(j)}^{i} \stackrel{\sim}{\longrightarrow} \mathcal{R}_{(j)}^{g-i+j}$, so in the proof of (i) we may further assume that $2 i-j \leq g$. We have

$$
\operatorname{dim}_{\mathbb{Q}}\left(R_{(j)}^{i}\right)=\sum_{k \leq i} \operatorname{dim}_{\mathbb{Q}}\left(M_{(j)}^{k}\right) .
$$

On the other hand, with $h \in \mathfrak{s I}_{2}$ as on p. 473 we have $\mathcal{R}_{(j)}^{i}=\left\{\alpha \in \mathcal{R}_{(j)} \mid h(\alpha)=\right.$ $(2 i-j-g) \cdot \alpha\}$. So it follows from (2) that

$$
\operatorname{dim}_{\mathbb{Q}}\left(\mathcal{R}_{(j)}^{i}\right)=\sum_{k \leq i} \mu(k, j)
$$

Part (i) now follows from the remark that $\mu(k, j)=\operatorname{dim}\left(M_{(j)}^{k}\right)$ if $k \leq i$ and $i+j \leq g$, because then $M_{(j)}^{g-k+j+1}=0$. (As remarked in $2.5, M_{(j)}^{b}=0$ if 
$b>2 j$.) The first assertion of (ii) follows from (i), since the inequalities $j \leq g / 3$ and $2 i-j \leq g$ imply that $i+j \leq g$. Hence $\mu(i, j)=\operatorname{dim}_{\mathbb{Q}}\left(M_{(j)}^{i}\right)$ for all indices $i$ that occur in the right hand side of (2), and as we have seen in 2.5 this is the number of ordered partitions of $j$ with $i-j$ parts.

The previous corollaries can be interpreted as non-vanishing results. The picture that emerges is that for small $j$ there are no relations in $R_{(j)}$. By contrast, for large $j$ we expect many relations. We always have $\mathcal{R}_{(j)}=0$ for $j \geq g-1$, and Polishchuk's result about the vanishing of the class of $x_{j}$ for $j \geq \frac{g}{2}+1$ (see [15], Corollary 0.2) gives that $\mathcal{R}_{(g-2)}=0$ for all $g \geq 4$ and $\mathcal{R}_{(g-3)}=0$ for all $g \geq 6$. We expect that for a given $j$ there is a bound $G_{j}$ such that $\mathcal{R}_{(j)}=$ for all $g \geq G_{j}$. In fact, the conjecture of van der Geer and Kouvidakis - see 2.13 below - predicts that $\mathcal{R}_{(g-2 l)}=0$ for all $g \geq(l+1)^{2}$ and $\mathcal{R}_{(g-2 l+1)}=0$ for all $g \geq l(l+1)$. The following result gives a proof of this in the first non-trivial cases. The bounds we obtain are sharp; see the examples in 2.12 .

2.11 Corollary. For all $g \geq 9$ we have $\mathcal{R}_{(g-4)}=0$. For all $g \geq 12$ we have $\mathcal{R}_{(g-5)}=0$.

Proof. For the first assertion, assume $g \geq 9$. By [15] we already know that $p_{g-3}=0$; this means that $\operatorname{Sym}^{2}(\mathrm{St})$ does not occur in $\mathcal{R}_{(g-4)}$. Hence to prove that $\mathcal{R}_{(g-4)}=0$ it suffices, by the theorem, to show that the map $\mathscr{D}: M_{(g-4)}^{g-1} \rightarrow M_{(g-4)}^{g-2}$ is surjective. A basis for $M_{(g-4)}^{g-1}\left(\operatorname{resp} . M_{(g-4)}^{g-2}\right)$ is the set $\operatorname{Mon}_{(g-4)}^{g-1}\left(\right.$ resp. $\left.\operatorname{Mon}_{(g-4)}^{g-2}\right)$, which is in bijection with the set of partitions of $g-4$ with 3 (resp. 2) parts.

For $g=9$ the map

$$
\mathscr{D}: M_{(5)}^{8}=\mathbb{Q} \cdot x_{2}^{2} x_{4} \oplus \mathbb{Q} \cdot x_{2} x_{3}^{2} \longrightarrow M_{(5)}^{7}=\mathbb{Q} \cdot x_{2} x_{5} \oplus \mathbb{Q} \cdot x_{3} x_{4}
$$

is given by the matrix $\left(\begin{array}{cc}30 & 20 \\ 6 & 20\end{array}\right)$; so it is surjective. Similarly, for $g=10$ the map $\mathscr{D}: M_{(6)}^{9} \rightarrow M_{(6)}^{8}$ is given, for the natural monomial bases of the spaces involved, by the matrix

$$
\left(\begin{array}{ccc}
42 & 35 & 0 \\
6 & 15 & 60 \\
0 & 10 & 0
\end{array}\right)
$$

which has full rank.

Assuming now that $g \geq 11$ we have the relations

$$
\begin{aligned}
\mathscr{D}\left(x_{2}^{2} x_{g-5}\right) & =(g-3)(g-4) x_{2} x_{g-4}+6 x_{3} x_{g-5}, \\
\mathscr{D}\left(x_{2} x_{3} x_{g-6}\right) & =\left(\begin{array}{c}
g-3 \\
3
\end{array}\right) x_{2} x_{g-4}+\left(\begin{array}{c}
g-4 \\
2
\end{array}\right) x_{3} x_{g-5}+10 x_{4} x_{g-6},
\end{aligned}
$$


Vol. 84 (2009)

$$
\begin{aligned}
\mathscr{D}\left(x_{2} x_{4} x_{g-7}\right) & =\left(\begin{array}{c}
g-3 \\
4
\end{array}\right) x_{2} x_{g-4}+\left(\begin{array}{c}
g-5 \\
2
\end{array}\right) x_{4} x_{g-6}+15 x_{5} x_{g-7}, \\
\mathscr{D}\left(x_{3}^{2} x_{g-7}\right) & =2 \cdot\left(\begin{array}{c}
g-4 \\
3
\end{array}\right) x_{3} x_{g-5}+20 x_{5} x_{g-7} .
\end{aligned}
$$

One verifies by direct calculation that these elements are linearly independent; hence $x_{2} x_{g-4}, x_{3} x_{g-5}, x_{4} x_{g-6}$ and $x_{5} x_{g-7}$ are all in the image of $\mathscr{D}: M_{(g-4)}^{g-1} \rightarrow M_{(g-4)}^{g-2}$. But this image also contains $\mathscr{D}\left(x_{2} x_{i} x_{g-3-i}\right)$ for all $i$ with $2 \leq i \leq \frac{g-3}{2}$, and this element is a linear combination with positive coefficients of $x_{2} x_{g-4}, x_{i} x_{g-2-i}$ and $x_{i+1} x_{g-3-i}$. Using induction on $i$ this gives the desired surjectivity of $\mathscr{D}$.

For the second assertion of the corollary, assume $g \geq 12$. This time we need to show that $\mathscr{D}^{2}: M_{(g-5)}^{g-3} \rightarrow M_{(g-5)}^{g-1}$ is surjective.

By direct calculation we find the relations

$$
\begin{aligned}
& \mathscr{D}^{2}\left(x_{2}^{3} x_{g-7}\right)=36 \cdot\left(\begin{array}{c}
g-3 \\
4
\end{array}\right) x_{2} x_{g-5}+36 \cdot\left(\begin{array}{c}
g-5 \\
2
\end{array}\right) x_{3} x_{g-6} \\
& +180 x_{4} x_{g-7} \text {, } \\
& \mathscr{D}^{2}\left(x_{2}^{2} x_{3} x_{g-8}\right)=40 \cdot\left(\begin{array}{c}
g-3 \\
5
\end{array}\right) x_{2} x_{g-5}+3 g \cdot\left(\begin{array}{c}
g-5 \\
3
\end{array}\right) x_{3} x_{g-6} \\
& +20(g-6)(g-7) x_{4} x_{g-7}+420 x_{5} x_{g-8} \text {, } \\
& D^{2}\left(x_{2} x_{3}^{2} x_{g-9}\right)=40 \cdot\left(\begin{array}{c}
g-3 \\
6
\end{array}\right) x_{2} x_{g-5}+40 \cdot\left(\begin{array}{c}
g-4 \\
5
\end{array}\right) x_{3} x_{g-6} \\
& +40 \cdot\left(\begin{array}{c}
g-6 \\
3
\end{array}\right) x_{4} x_{g-7}+40 \cdot\left(\begin{array}{c}
g-7 \\
2
\end{array}\right) x_{5} x_{g-8} \\
& +1120 x_{6} x_{g-9} \text {, } \\
& \mathscr{D}^{2}\left(x_{2}^{2} x_{4} x_{g-9}\right)=60 \cdot\left(\begin{array}{c}
g-3 \\
6
\end{array}\right) x_{2} x_{g-5}+12 \cdot\left(\begin{array}{c}
g-5 \\
4
\end{array}\right) x_{3} x_{g-6} \\
& +12 \cdot\left(\begin{array}{c}
g-5 \\
4
\end{array}\right) x_{4} x_{g-7}+60 \cdot\left(\begin{array}{c}
g-7 \\
2
\end{array}\right) x_{5} x_{g-8} \\
& +840 x_{6} x_{g-9} \text {, }
\end{aligned}
$$




$$
\begin{aligned}
& \mathscr{D}^{2}\left(x_{2} x_{3} x_{4} x_{g-10}\right)= 70 \cdot\left(\begin{array}{c}
g-3 \\
7
\end{array}\right) x_{2} x_{g-5}+30 \cdot\left(\begin{array}{c}
g-4 \\
6
\end{array}\right) x_{3} x_{g-6} \\
&+ 4 g \cdot\left(\begin{array}{c}
g-6 \\
4
\end{array}\right) x_{4} x_{g-7}+30 \cdot\left(\begin{array}{c}
g-7 \\
3
\end{array}\right) x_{5} x_{g-8} \\
&+70 \cdot\left(\begin{array}{c}
g-8 \\
2
\end{array}\right) x_{6} x_{g-9}+2520 x_{7} x_{g-10} \\
& D^{2}\left(x_{3}^{3} x_{g-10}\right)=120 \cdot\left(\begin{array}{c}
g-4 \\
6
\end{array}\right) x_{3} x_{g-6}+120 \cdot\left(\begin{array}{c}
g-7 \\
3
\end{array}\right) x_{5} x_{g-8} \\
&+3360 x_{7} x_{g-10} .
\end{aligned}
$$

One checks that these elements span the whole space

$\mathbb{Q} \cdot x_{2} x_{g-5}+\mathbb{Q} \cdot x_{3} x_{g-6}+\mathbb{Q} \cdot x_{4} x_{g-7}+\mathbb{Q} \cdot x_{5} x_{g-7}++\mathbb{Q} \cdot x_{6} x_{g-9}+\mathbb{Q} \cdot x_{7} x_{g-10}$,

so this subspace of $M_{(g-5)}^{g-1}$ is fully contained in the image of $\mathscr{D}^{2}: M_{(g-5)}^{g-3} \rightarrow M_{(g-5)}^{g-1}$.

On the other hand, for every index $i$ with $2<i<\frac{g-5}{2}$ this image also contains the element $\mathscr{D}^{2}\left(x_{2}^{2} x_{i} x_{g-5-i}\right)$, which is a linear combination with positive coefficients of the elements $x_{2} x_{g-5}, x_{3} x_{g-6}, x_{i} x_{g-3-i}, x_{i+1} x_{g-4-i}$ and $x_{i+2} x_{g-5-i}$. From this it follows by induction on $i$ that $\mathscr{D}^{2}$ surjects to $M_{(g-5)}^{g-1}$, as claimed.

2.12 Examples. In Figure 2 we give the dimensions of all $\mathcal{R}_{(j)}^{i}$ for some low genera. The numbering scheme is the same as in Figure 1, but we omit the part with negative level. If in box $(i, j)$ a number $d$ appears, this means that $\operatorname{dim}_{\mathbb{Q}}\left(\mathcal{R}_{(j)}^{i}\right)=d$. The unnumbered boxes correspond to the summands $\mathcal{R}_{(j)}^{i}$ that are zero.

Note that for $g=10$ there is a mistake in Polishchuk's list of relations in [15], Section 2.6. It is not true that $x_{3} x_{4}$ and $x_{2} x_{5}$ are both zero, as stated there; we only have the relation $3 x_{3} x_{4}+7 x_{2} x_{5}=0$.

Though these tables only give the dimensions of the spaces $\mathcal{R}_{(j)}^{i}$, it should be clear that with little extra work one can actually write down a basis. Alternatively, using Remark 2.7 (ii) one can give a basis for $\mathcal{R}_{(j)}$ consisting of primitive elements.

2.13. Gerard van der Geer and Alexis Kouvidakis have informed me (personal communication of van der Geer) that they have a conjecture for the dimension of the spaces $\mathcal{R}_{(j)}^{i}$. Before we state their conjecture, let us introduce the notation $p_{\mathbf{x}}(n ; \mathbf{y})$ for the number of partitions of $n$ with conditions $\mathbf{x}$ on the number of parts, and conditions $\mathbf{y}$ on the parts. If we impose no condition, we omit $\mathbf{x}$ or $\mathbf{y}$ from the notation. Thus, for instance, by $p_{k}(n ; \leq l)$ we mean the number of partitions of $n$ 

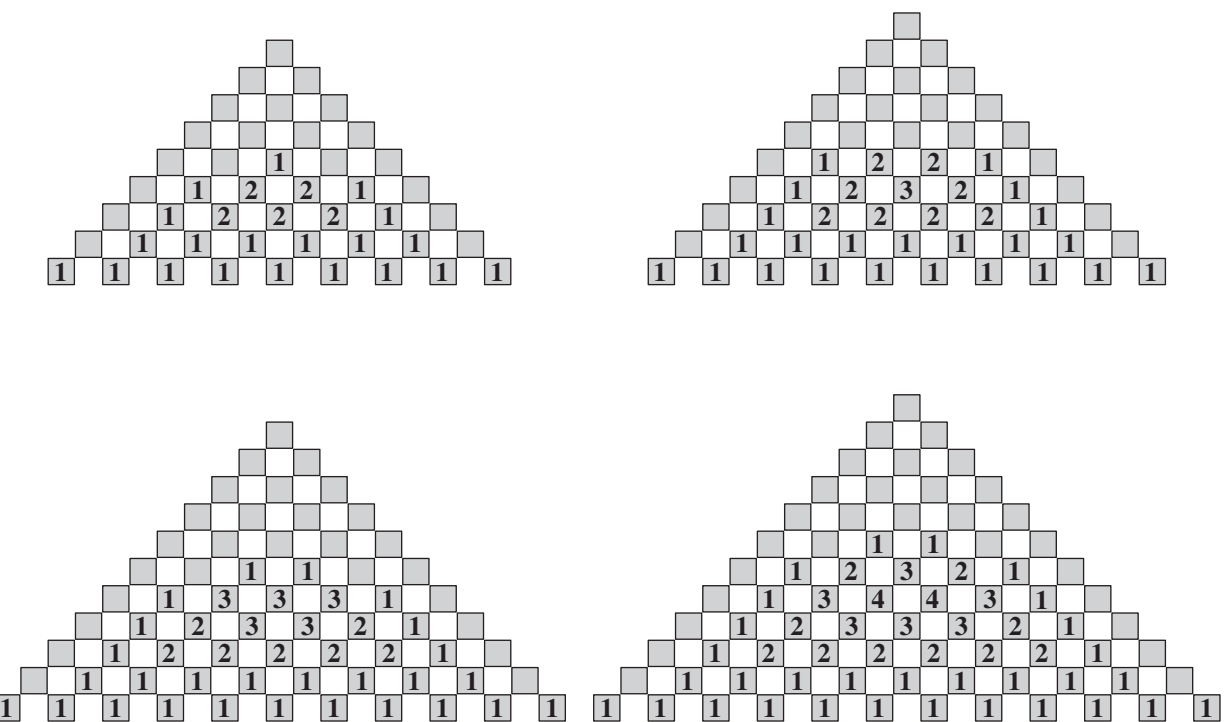

$\begin{array}{lllllllllllllllllllllllllll}1 & 1 & 1 & 1 & 1 & 1 & 1 & 1 & 1 & 1 & 1 & 1 & 1 & 1 & 1 & 1 & 1 & 1 & 1 & 1 & 1 & 1 & 1\end{array}$

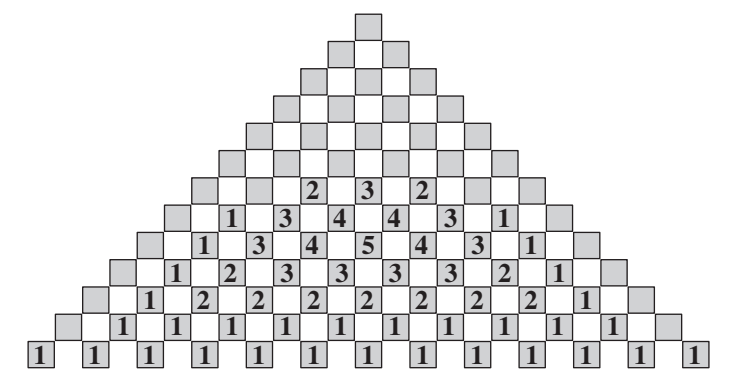

Figure 2. The structure of the ring $\mathcal{R}$ for some low values of $g$.

with $k$ parts and with all parts $\leq l$. Conjugation of partitions interchanges the conditions " $\mathbf{x}$ " and " $\mathbf{y}$ "; for instance, $p_{k}(n ; \leq l)=p_{\leq l}(n ; \max =k)$. Also note that $p_{\leq k}(n ; \leq l)=p_{k}(n+k ; \leq l+1)$.

The conjecture of van der Geer and Kouvidakis is that $\operatorname{dim}_{\mathbb{Q}}\left(\mathcal{R}_{(j)}^{i}\right)$ equals $p_{i-j}(i ; \leq g+1-i)$, the number of partitions of $i$ with $i-j$ parts and all parts at most $g+1-i$. We make the convention that $p_{0}(0 ; g+1)=1$. They have verified their conjecture for all $g$ up to 25 . Using the remarks just made, we see that the conjecture is compatible with Fourier duality.

Note that the conjecture gives that the dimension of $\mathcal{R}^{i}$ equals $p(i ; \leq g+1-i)=$ $p_{\leq g+1-i}(i)=p_{g+1-i}(g+1)$, and that the dimension of $\mathcal{R}$ equals $p(g+1)$, the number of partitions of $g+1$. 
Van der Geer and Kouvidakis also have a strong version of the conjecture, namely that the monomials $x^{\alpha}$ corresponding to the permutations of $i$ with $i-j$ parts and all parts $\leq g+1-i$ give a basis for $\mathcal{R}_{(j)}^{i}$.

Though I do not know a proof for the conjecture, it is interesting to compare it with Theorem 2.6. Namely, if $2 i-j \leq g$ the conjecture gives, after some rewriting, that the representation $\mathrm{Sym}^{g-2 i+j}(\mathrm{St})$ occurs in $\mathcal{R}_{(j)}$ with multiplicity

$$
p_{i-j}(j ; \leq g-i)-p_{g+1-i}(j ; \leq i-j-1) .
$$

The correctness of this formula suffices to prove the conjecture. Note that in our calculation of this multiplicity as the corank of the map $\mathscr{D}^{g-2 i+j+1}: M_{(j)}^{g-i+j+1} \rightarrow$ $M_{(j)}^{i}$, the source space has dimension $p_{g+1-i}(j)$ and the target space has dimension $p_{i-j}(j)$.

In a first version of this paper, I had suggested that the maps $\mathscr{D}^{g-2 i+j+1}$ always have the maximum possible rank. This turns out not to be true. The first counterexample occurs for $g=17$, with $i=12$ and $j=10$; in that case we look at $D^{4}: M_{(10)}^{16} \rightarrow M_{(10)}^{12}$, in which source and target are both 5-dimensional, but which has rank only 4.

As pointed out to me by Gerard van der Geer, the conjecture fits nicely with BrillNoether theory. Namely, if we fix $r=i-j$ and look for the smallest $j$ such that $\mathcal{R}_{(j)}^{j+r}=0$ then the predicted result is exactly what could be expected from geometry. To be precise, given $g$ and $r$, let $d(g, r)=g+r-\left\lfloor\frac{g}{r+1}\right\rfloor$ be the smallest positive integer $d$ such that the general curve of genus $g$ has a $g_{d}^{r}$. By Theorem 2.14 below, having a $g_{d}^{r}$ gives a relation in $\mathcal{R}_{(j)}^{j+r}$ for all $j \geq d-2 r+1$. Now observe that the conjecture of van der Geer and Kouvidakis predicts that $\mathcal{R}_{(j)}^{j+r}=0$ if and only if $j \geq d(g, r)-2 r+1$. For $r=1$ this is Polishchuk's result on the vanishing of the classes $p_{n}$ for $n \geq \frac{g}{2}+1$. For other small $r$, say $r=2$ and 3, this can be proven with arguments as in 2.11, but as it seems difficult to get a general result with this method, we shall not give the details here.

In the remainder of this section, we briefly want to recall some finer results on the structure of the tautological subring of $A(J)$ if we assume that the curve admits a linear system of a given rank and dimension. The following result was proven by Herbaut in [10] in a different formulation. Soon thereafter the result as stated here was proven by van der Geer and Kouvidakis in [9]. It was shown by Zagier in an appendix to [9] that the two results are actually equivalent. We shall further refine this result in Section 4. See [14] for another proof.

2.14 Theorem (Herbaut [10], van der Geer-Kouvidakis [9]). If the curve $C$ has a $g_{d}^{r}$ then in $A(J)$, for all $i>d-r$,

$$
\sum_{\substack{m_{1}, \ldots, m_{r} \\ m_{1}+\cdots+m_{r}=i}} m_{1} ! \ldots m_{r} ! \cdot p_{m_{1}} \ldots p_{m_{r}}=0 .
$$


2.15 Remark. The theorem generalizes the older result of Colombo and van Geemen [4] saying that if $C$ has a $g_{d}^{1}$ then $p_{i}=0$ in $A(J)$ for all $i \geq d$. In an earlier version of this paper, the author had a (conditional) result, very similar to [10], Theorem 4, about the vanishing of the classes $p_{i}$ for $i \geq d-2 r+2$ in case the curve has a $g_{d}^{r}$. It was pointed out to me by the referee that such a result actually follows from the theorem of Colombo and van Geemen by using the results of Coppens and Martens in [5]. Indeed, if $c$ is the Clifford index of $C$, which by definition is the minimum of $d-2 r$ over all special $g_{d}^{r}$ on the curve, then it is shown in [5] that $C$ admits either a $g_{c+2}^{1}$ or a $g_{c+3}^{1}$. Further, the curves $C$ that do not admit a $g_{c+2}^{1}$ are exceptional; see Eisenbud et al. in [6]. So indeed the existence of a $g_{d}^{r}$ on $C$ implies that $p_{i}=0$ in $A(J)$ for all $i \geq d-2 r+3$ and even $i \geq d-2 r+2$ except when $C$ is of a very particular type.

2.16. The general pattern we see is that the existence of special divisors on $C$ leads to relations between the generators $p_{n}$ of the tautological ring. One may ask if there is a converse to this. See for instance Herbaut [10], just after Theorem 4. However, it seems to us that one cannot expect such a converse, at least not in a naive way. The point is that for varieties over $\overline{\mathbb{Q}}$ one expects that the filtration $\mathrm{Fil}^{\bullet} \mathrm{CH}(J)$ introduced in 1.2 satisfies $\mathrm{Fil}^{2}=0$. In particular, for curve $C$ over $\overline{\mathbb{Q}}$ we should have $p_{n}=0$ for all $n \geq 3$. On the other hand, the general curve of genus $g$ over $\overline{\mathbb{Q}}$ is also general in the sense of Brill-Noether theory.

As yet this is of course only speculation, but it leads to the interesting question whether one can obtain relations between the classes $p_{n}$ from the assumption that there is a non-constant map $C \rightarrow \mathbb{P}^{1}$ with at most three critical values.

\section{The big tautological ring}

3.1. In the rest of the paper we shall be interested in cycle relations in the Chow ring of $J$ (tensored with $\mathbb{Q}$; see our conventions on p. 473). Note that from now on we will consider an operator $\mathscr{D}$ on $\mathrm{CH}(J)$ that lifts the operator considered before. Also we will consider elements $p_{n}$ that lift those defined in 2.2.

3.2 Definition. We define the big tautological ring Taut $(C) \subset \mathrm{CH}(J)$ as the smallest $\mathbb{Q}$-subalgebra of $\mathrm{CH}(J)$ that contains the image of $j_{*}: \mathrm{CH}(C) \rightarrow \mathrm{CH}(J)$ and is stable under all operations $\cdot, *, \mathcal{F}, n^{*}$ and $n_{*}$. Similarly, the small tautological ring $\operatorname{taut}(C) \subset \mathrm{CH}(J)$ is the smallest $\mathbb{Q}$-subalgebra of $\mathrm{CH}(J)$ that contains the classes $[C]_{(j)}$ and is stable under all operations $\cdot, *, \mathscr{F}, n^{*}$ and $n_{*}$.

The small and big tautological rings have the same image in $A(J):=\mathrm{CH}(J) / \sim_{\text {alg }}$; this image is the tautological ring $\mathcal{T}(C) \subset A(J)$ considered before. In general, 
Taut $(C)$ is much bigger than $\operatorname{taut}(C)$. To make this more precise we first recall that by the results of [16], to be briefly reviewed below, $\operatorname{taut}(C)$ is finitely generated. By contrast, Taut $(C)$ contains the whole $\mathrm{CH}^{g}(J)$; hence it also contains all $\mathrm{CH}_{(i)}^{i}$.

3.3 Remark. The big tautological ring $\operatorname{Taut}(C) \subset \mathrm{CH}(J)$ does not depend on the choice of the base point $x_{0} \in C$. To see this, let $x_{0}^{\prime} \in C$ be another base point, let $j^{\prime}: C \hookrightarrow J$ be the associated embedding, and let $\operatorname{Taut}^{\prime}(C)$ be the resulting tautological ring. We have $j^{\prime}=t_{\delta} \circ j$ with $\delta=\left[O_{C}\left(x_{0}-x_{0}^{\prime}\right)\right] \in J$. Note that the class $[\delta] \in \mathrm{CH}^{g}(J)$ lies in $\operatorname{Taut}(C)$, as $[\delta]=(-1)^{*} j_{*}\left(\left[x_{0}^{\prime}\right]\right)$. For $y \in \mathrm{CH}(C)$ we have the relation $j_{*}^{\prime}(y)=[\delta] * j_{*}(y)$. So $j_{*}^{\prime}(C) \subset$ Taut $(C)$, and it follows that $\operatorname{Taut}^{\prime}(C) \subseteq \operatorname{Taut}(C)$. Hence, by symmetry, $\operatorname{Taut}(C)=\operatorname{Taut}^{\prime}(C)$.

3.4. Let $\theta \in \mathrm{CH}^{1}(J)$ be the class of a symmetric theta divisor. As in [16] we define classes $p_{n} \in \mathrm{CH}_{(n-1)}^{n}(J)$ and $q_{n} \in \mathrm{CH}_{(n)}^{n}(J)$ by

$$
\begin{array}{ll}
p_{n}:=\mathcal{F}\left([j(C)]_{(n-1)}\right)=\text { degree } n \text { component of } \mathcal{F}[j(C)], & 1 \leq n \leq g ; \\
q_{n}:=\mathscr{F}\left(\theta \cdot[j(C)]_{(n)}\right)=\text { degree } n \text { component of } \mathcal{F}(\theta \cdot[j(C)]), & 0 \leq n \leq g-1 .
\end{array}
$$

In particular we have $p_{1}=-\theta$ and $q_{0}=g$. We set $p_{n}:=0$ if $n \leq 0$ or $n>g$ and $q_{n}:=0$ if $n<0$ or $n \geq g$.

If $y$ is an element of $\mathrm{CH}(C)$, write

$$
a_{n}(y):=\mathcal{F}\left(\left(j_{*} y\right)_{(n)}\right) .
$$

If $y \in \mathrm{CH}^{1}(C)$ then $a_{n}(y)$ is the degree $n$ component of $\mathcal{F}\left(j_{*} y\right)$, which lies in $\mathrm{CH}_{(n)}^{n}(J)$. If $D$ is a divisor on $C$ then we write $a_{n}(D):=a_{n}([D])$. Note that, with $K$ the canonical class of $C$, we have $\theta \cdot[j(C)]=\frac{1}{2} j_{*} K+[0]$; see [16], Section 1 . Hence

$$
q_{n}= \begin{cases}g \cdot[J]=[J]+\frac{1}{2} a_{0}(K) & \text { if } n=0 \\ \frac{1}{2} a_{n}(K) & \text { if } n \neq 0 .\end{cases}
$$

3.5. Write $\ell:=c_{1}\left(\mathscr{L}_{J}\right)$, with $\mathscr{L}_{J}$ as in 1.1. As in [16] we define, for $n \in \mathbb{Z}_{\geq 0}$ and $a \in \mathrm{CH}(J)$, an operator $A_{n}(a) \in \operatorname{End}(\mathrm{CH}(J))$ by $A_{n}(a)(b)=a *_{n} b:=$ $\left(p_{1}+p_{2}\right)_{*}\left(\ell^{n} \cdot p_{1}^{*} a \cdot p_{2}^{*} b\right)$. For $n<0$ we set $A_{n}(a):=0$.

Let $y$ be an element of $\mathrm{CH}(C)$. Given integers $m \geq 0$ and $n \geq 0$ we define $Z_{m, n}(y):=m ! \cdot A_{n}\left(\left(j_{*} y\right)_{(m+n)}\right)$. If $m<0$ or $n<0$ we set $Z_{m, n}(y):=0$. If $D$ is a divisor on $C$ we write $Z_{m, n}(D):=Z_{m, n}([D])$.

The operators $Y_{m, n}$ considered in [16] are given by

$$
Y_{m, n}:= \begin{cases}\mathrm{id}+\frac{1}{2} Z_{0,0}(K)=g \cdot \mathrm{id} & \text { if }(m, n)=(0,0) \\ \frac{1}{2} Z_{m, n}(K) & \text { else. }\end{cases}
$$


Further, Polishchuk introduces operators $\tilde{X}_{m, n}$ and $X_{m, n}$ given by

$$
\tilde{X}_{m, n}:=m ! \cdot A_{n}\left([C]_{(m+n-2)}\right) \quad \text { and } \quad X_{m, n}:=\tilde{X}_{m, n}-m n Y_{m-1, n-1} .
$$

Note that the $X_{m, n}$ and $\tilde{X}_{m, n}$ can be non-zero only if $m+n \geq 2$. If $m<0$ or $n<0$ then we set $Y_{m, n}=\tilde{X}_{m, n}=X_{m, n}:=0$.

The first main result of [[16] is that we have the commutation relations

$$
\begin{aligned}
{\left[X_{m, n}, X_{m^{\prime}, n^{\prime}}\right] } & =\left(m^{\prime} n-m n^{\prime}\right) \cdot X_{m+m^{\prime}-1, n+n^{\prime}-1}, \\
{\left[X_{m, n}, Y_{m^{\prime}, n^{\prime}}\right] } & =\left(m^{\prime} n-m n^{\prime}\right) \cdot Y_{m+m^{\prime}-1, n+n^{\prime}-1}, \\
{\left[Y_{m, n}, Y_{m^{\prime}, n^{\prime}}\right] } & =0,
\end{aligned}
$$

where in the second line we restrict to elements $X_{m, n}$ with $m+n \geq 2$. These identities can be interpreted as saying that a suitable Lie algebra acts on $\mathrm{CH}(J)$.

The triple $\left(X_{2,0} / 2, X_{1,1}, X_{0,2} / 2\right)$ defines the action of $\mathfrak{s}_{2}$ on $\mathrm{CH}(J)$ as in 1.3. The operator $\mathscr{D}:=\frac{1}{2} X_{2,0}$ will play an important role in what follows; note that it is given by $\mathscr{D}(x)=[C]_{(0)} * x$ and that it maps $\mathrm{CH}_{(j)}^{i}(J)$ to $\mathrm{CH}_{(j)}^{i-1}(J)$.

The second main result of [16] is that $\operatorname{taut}(C)$ is generated by the classes $p_{m}$ $(1 \leq m \leq g)$ and $q_{m}(0 \leq m \leq g-1)$, and that $\mathscr{D}$ acts on it as the differential operator

$$
\begin{aligned}
& \frac{1}{2} \cdot \sum_{m, n \geq 1}\left(\begin{array}{c}
m+n \\
n
\end{array}\right) p_{m+n-1} \partial_{p_{m}} \partial_{p_{n}} \\
& \quad+\sum_{m, n \geq 1}\left(\begin{array}{c}
m+n-1 \\
n
\end{array}\right) q_{m+n-1} \partial_{q_{m}} \partial_{p_{n}}-\sum_{m \geq 1} q_{m-1} \partial_{p_{m}} .
\end{aligned}
$$

(Polishchuk also has explicit formulas for the other operators $X_{m, n}$ and $Y_{m, n}$; for these we refer to [16].) Note that if we say that $\mathscr{D}$ acts as the operator (5), the formal meaning is that we consider the polynomial ring $\mathbb{Q}\left[\left\{p_{m}\right\}_{1 \leq m \leq g},\left\{q_{m}\right\}_{0 \leq m \leq g-1}\right]$, where we now view the $p_{m}$ and $q_{m}$ as indeterminates; then the natural homomorphism $\mathbb{Q}\left[p_{\bullet}, q_{\bullet}\right] \rightarrow \operatorname{taut}(C) \subset \mathrm{CH}(J)$ intertwines the differential operator given by (5) and the operator $\mathscr{D}$.

The main purpose of this section is to extend Polishchuk's results to the big tautological ring, as follows.

3.6 Theorem. (i) The big tautological ring Taut $(C)$ is generated, as a $\mathbb{Q}$-algebra, by the classes $p_{m}$ and $a_{m}(D)$, for $m \geq 0$ and $D$ a divisor on $C$.

(ii) Let $D_{1}, \ldots, D_{s}$ be divisors on $C$, and let $R$ be the $\mathbb{Q}$-subalgebra of Taut $(C)$ generated by the classes $p_{m}, q_{m}$ and $a_{m}\left(D_{i}\right)$ for $m \geq 0$ and $i \in\{1, \ldots, s\}$. Then $R$ is stable under the operations $; *, \mathcal{F}, n^{*}, n_{*}$ and $\mathscr{D}$, and $\mathscr{D}$ acts on it as the 
differential operator

$$
\begin{aligned}
& \frac{1}{2} \cdot \sum_{m, n \geq 1}\left(\begin{array}{c}
m+n \\
n
\end{array}\right) p_{m+n-1} \partial_{p_{m}} \partial_{p_{n}}+\sum_{m, n \geq 1}\left(\begin{array}{c}
m+n-1 \\
n
\end{array}\right) q_{m+n-1} \partial_{q_{m}} \partial_{p_{n}} \\
& +\sum_{i=1}^{s} \sum_{m, n \geq 1}\left(\begin{array}{c}
m+n-1 \\
n
\end{array}\right) a_{m+n-1}\left(D_{i}\right) \partial_{a_{m}\left(D_{i}\right)} \partial_{p_{n}}-\sum_{m \geq 1} q_{m-1} \partial_{p_{m}} .
\end{aligned}
$$

For the proof of this result we will closely follow the arguments of [16].

3.7 Lemma. Let $D$ be a divisor on $C$. Then for any $n \geq 0$ we have $Z_{0, n}(D)(x)=$ $n ! \cdot a_{n}(D) \cdot x$.

For the proof we refer to [16], Lemma 2.4.

3.8 Proposition. We have the commutation relations

(a) $\left[Z_{m, n}(D), Z_{m^{\prime}, n^{\prime}}\left(D^{\prime}\right)\right]=0$,

(b) $\left[X_{m, n}, X_{m^{\prime}, n^{\prime}}\right]=\left(n m^{\prime}-m n^{\prime}\right) \cdot X_{m+m^{\prime}-1, n+n^{\prime}-1}$,

(c) $\left[X_{m, n}, Z_{m^{\prime}, n^{\prime}}(D)\right]=\left(n m^{\prime}-m n^{\prime}\right) \cdot Z_{m+m^{\prime}-1, n+n^{\prime}-1}(D)$,

where in (c) we restrict to elements $X_{m, n}$ with $m+n \geq 2$.

Proof. Relations (a) and (b) were proved in [16]; see loc. cit., Theorem 0.1 and the remark following Lemma 2.5. Part (c) can be proven by the same arguments as in the proof of [16], Theorem 2.6. Instead of giving full details, let us note that (c) also follows from [17], Theorem 2.1. To make the connection, let us first note that, since we work over a field, the term $\psi$ that appears in Polishchuk's relation is zero, and also all terms $p_{0}^{*}(a)$ vanish. (Note that $g>1$.) Hence, taking $a=C$ and $a^{\prime}=[D]$, loc. cit., Theorem 2.1 reads

$$
\left[T_{k}(m, C), T_{k^{\prime}}\left(m^{\prime},[D]\right)\right]=\left(k m^{\prime}-k^{\prime} m\right) \cdot T_{k+k^{\prime}-1}\left(m+m^{\prime},[D]\right),
$$

where

$$
T_{k}(m, C)=\sum_{n \geq 0} \frac{m^{n}}{n !} \tilde{X}_{n, k} \quad \text { and } \quad T_{k^{\prime}}\left(m^{\prime},[D]\right)=\sum_{\nu \geq 0} \frac{\left(m^{\prime}\right)^{v}}{\nu !} Z_{v, k^{\prime}}(D) .
$$

As (7) holds for all $m$ we obtain, using (a), that

$$
\left[X_{n, k}, Z_{v, k^{\prime}}(D)\right]=\left[\tilde{X}_{n, k}, Z_{v, k^{\prime}}(D)\right]=\left(k v-k^{\prime} n\right) \cdot Z_{n+v-1, k+k^{\prime}-1}(D),
$$

which is relation (c). 
3.9. Proof of Theorem 3.6. Let $D_{1}, \ldots, D_{s}$ and $R$ be as in (ii) of the theorem. Consider the polynomial ring

$$
\widetilde{R}:=\mathbb{Q}\left[\left\{p_{m}\right\}_{m \geq 1},\left\{q_{m}\right\}_{m \geq 0},\left\{a_{m}\left(D_{v}\right)\right\}_{m \geq 0,1 \leq v \leq s}\right]
$$

and let $\pi: \widetilde{R} \rightarrow R$ be the natural map. Define $\widetilde{D}$ to be the differential operator on $\widetilde{R}$ given by (6).

We first prove that $R$ is stable under $\mathscr{D}$ and that the action of $\mathscr{D}$ is given by (6), in other words, $\pi \circ \widetilde{D}=\mathscr{D} \circ \pi$. The first step in the proof is to show that if $x$ and $y$ are any of the variables $p_{m}, q_{m}$ or $a_{m}\left(D_{v}\right)$ then

$$
\pi \circ[[\tilde{D}, x], y]=[[\mathscr{D}, x], y] \circ \pi ;
$$

here " $x$ " stands for "multiplication by $x$ " on $\widetilde{R}$ (resp. on $R$ ), and likewise for " $y$ ". Note that if (8) holds for the pair $(x, y)$ then it also holds for $(y, x)$, as follows from the Jacobi identity, using that $[x, y]=0$. It follows from Polishchuk's results in [16] that

$$
\pi \circ\left[\tilde{D}, p_{m}\right]=\left[\mathscr{D}, p_{m}\right] \circ \pi
$$

for all $m$, as $\widetilde{D}$ is the sum of the operator given by (5) and an operator that commutes with $p_{m}$. Because of the relation (4), it only remains to verify (8) for elements $x=a_{m}\left(D_{i}\right)$ and $y=a_{n}\left(D_{j}\right)$. Direct calculation gives $\left[\left[\widetilde{\mathscr{D}}, a_{m}\left(D_{i}\right)\right], a_{n}\left(D_{j}\right)\right]=$ 0. On the other hand, using Lemma 3.7 and Proposition 3.8 we find that also $\left[\left[\mathscr{D}, a_{m}\left(D_{i}\right)\right], a_{n}\left(D_{j}\right)\right]=0$. Hence we have (8).

By induction on the degree it follows from (8) that for all $F \in \widetilde{R}$ we have

$$
\pi\left(\left[\widetilde{D}, a_{m}\left(D_{v}\right)\right](F)\right)=\left[\mathscr{D}, a_{m}\left(D_{v}\right)\right](\pi(F)) .
$$

Note that to start the induction we need to prove this relation for $F$ the unit element of $\widetilde{R}$, which maps to the class $[J]$ in $R$. Then the LHS of (10) is zero. The RHS equals $\left[\mathscr{D}, a_{m}\left(D_{v}\right)\right]([J])=\mathscr{D}\left(a_{m}\left(D_{v}\right)\right)-a_{m}\left(D_{v}\right) \cdot \mathscr{D}([J])$, which is zero, too, because $\mathscr{D}([J]) \in \mathrm{CH}^{-1}(J)=0$ and $\mathscr{D}\left(a_{m}\left(D_{v}\right)\right) \in \mathrm{CH}_{(m)}^{m-1}(J)=0$. Hence we can start the induction, and we get the relation (10). In particular, using (4) we find that

$$
\pi \circ\left[\tilde{D}, q_{m}\right]=\left[\mathscr{D}, q_{m}\right] \circ \pi .
$$

By another induction on the degree it follows from (9), (10) and (11) that $\pi(\widetilde{D}(F))=$ $\mathscr{D}(\pi(F))$ for all $F \in \widetilde{R}$, which is what we wanted to prove.

By definition $R \subset \mathrm{CH}(J)$ is stable under intersection product, and as just proven it is stable under $\mathscr{D}$. As the Fourier transform can be calculated as $\mathscr{F}=$ $\exp (e) \cdot \exp (-f) \cdot \exp (e)$ (see [16], end of Section 1, or [18], Lemma 1.4), $R$ is also stable under $\mathscr{F}$, and hence also under Pontryagin product. The generators of $R$ are homogeneous (for the usual grading by codimension), so $R$ is a graded subalgebra of $\mathrm{CH}(J)$, and because for $y \in \mathrm{CH}^{i}(J)$ we have $y \in \mathrm{CH}_{(j)}^{i}(J)$ if and only 
if $\mathcal{F}(y) \in \mathrm{CH}^{g-i+j}(J)$, we find that $R$ is stable under Beauville's decomposition, hence also under all $n^{*}$ and $n_{*}$. This proves (ii) of the theorem.

Finally, let $T^{\prime} \subset \operatorname{Taut}(C)$ be the $\mathbb{Q}$-subalgebra generated by all classes $p_{m}, q_{m}$ and $a_{m}(D)$. By the results just proven, $T^{\prime}$ is stable under all operators $\cdot, *, \mathcal{F}, n^{*}$ and $n_{*}$, and as $T^{\prime}$ contains the image of $j_{*}$ we conclude that $T^{\prime}=\operatorname{Taut}(C)$.

3.10 Corollary. Let $D$ be a divisor on $C$, and consider the $\mathbb{Q}$-subalgebra $R \subset$ Taut $(C)$ generated by the classes $p_{m}, q_{m}$ and $a_{m}(D)$. Then for all $m \geq 1+\frac{g}{2}$ the class $a_{m}(D)$ lies in the ideal of $R$ generated by the classes $q_{n}$ with $1 \leq n<\frac{g+1}{2}$.

Proof. Write $a_{m}:=a_{m}(D)$. Let $S \subset R$ be the subalgebra generated by the classes $p_{m}$ for $m \geq 2$, together with all $q_{m}$ and $a_{m}$. (So the only generator we exclude is $p_{1}$.) Then $S$ is stable under $\mathscr{D}$ and so is the ideal $I=S \cdot q_{1}+\cdots+S \cdot q_{g-1}$. The induced operator $\bar{D}$ on $S / I$ is given by

$$
\frac{1}{2} \cdot \sum_{m, n \geq 1}\left(\begin{array}{c}
m+n \\
n
\end{array}\right) p_{m+n-1} \partial_{p_{m}} \partial_{p_{n}}+\sum_{m, n \geq 1}\left(\begin{array}{c}
m+n-1 \\
n
\end{array}\right) a_{m+n-1} \partial_{a_{m}} \partial_{p_{n}} .
$$

First suppose $g$ is even. Consider the element $a_{1} p_{2}^{u}$ for some $u \geq 1$. By induction we find that $\bar{D}^{n}\left(a_{1} p_{2}^{u}\right)$, for $0 \leq n \leq u-1$, is a linear combination with coefficients in $\mathbb{Z}_{\geq 0}$ of terms $a_{k} p_{m_{1}} \ldots p_{m_{u-n}}$ such that $k+m_{1}+\cdots+m_{u-n}=2 u+1-n$. In particular, $\bar{D}^{u-1}\left(a_{1} p_{2}^{u}\right)$ is a linear combination of terms $a_{k} p_{m}$ with $k+m=u+2$, and again applying $\bar{D}$ gives $\bar{D}^{u}\left(a_{1} p_{2}^{u}\right)=c \cdot a_{u+1}$ for some positive integer $c$. (One readily sees that $c$ is non-zero.) Now use that for all $u \geq g / 2$ we have $a_{1} p_{2}^{u}=0$ in $S / I$.

Similarly, if $g$ is odd, we start with the relation $a_{1} p_{2}^{u} p_{3}=0$ for $u \geq \frac{g-1}{2}$, and applying $\bar{D}^{u}$ we obtain that $a_{u+2}=0$ in $S / I$.

This shows that $a_{m} \in I \cdot R$ for all $m \geq 1+\frac{g}{2}$. Finally use [16], Proposition 4.2, which tells us that already in the small tautological ring $\operatorname{taut}(C) \subset R$ the ideal $\left(q_{1}, \ldots, q_{g-1}\right)$ is generated by the classes $q_{n}$ with $n<\frac{g+1}{2}$.

\section{Cycle relations in the Chow ring}

4.1. As before, let $C$ be a complete non-singular curve of genus $g \geq 2$. Choose a base point $x_{0} \in C$, and let $\mathscr{L}$ be the Poincaré bundle on $C \times J$, normalised such that $\mathscr{L}_{\mid\left\{x_{0}\right\} \times J}$ is trivial.

From now on we assume $C$ has a complete base-point free $g_{d}^{r}$, say $|\Gamma|$. Write $V:=H^{0}(C, \Gamma)$, and let $\gamma: C \rightarrow \mathbb{P}\left(V^{\vee}\right) \cong \mathbb{P}^{r}$ be the morphism associated to the linear system. If there is no risk of confusion we simple write $\mathbb{P}:=\mathbb{P}(V)$. 
Consider the incidence variety

$$
Y:=\left\{(P, \ell) \in C \times \mathbb{P} \mid \ell \subset H^{0}(C, \Gamma-P)\right\},
$$

and let $q_{1}: Y \rightarrow C$ and $q_{2}: Y \rightarrow \mathbb{P}$ be the two projections. As in van der GeerKouvidakis [9] we want to apply Grothendieck-Riemann-Roch to the line bundle $\mathcal{M}:=\left(q_{1} \times \text { id }\right)^{*} \mathscr{L}$ on $Y \times J$ and the morphism $\left(q_{2} \times\right.$ id $): Y \times J \rightarrow \mathbb{P} \times J$. Our main task is to refine their calculations so as to make them work on Chow level.

4.2 Proposition. We have

$$
\begin{aligned}
\operatorname{ch}\left(\left(q_{2} \times \mathrm{id}\right)_{*} \mathcal{M}\right) & =\mathcal{F}\left(j_{*}\left([C]-\frac{1}{2} K\right)\right)-\mathcal{F}\left(j_{*}\left([C]-\frac{1}{2} K-[\Gamma]\right)\right) \cdot \exp (-h), \\
& =\mathcal{F}\left(j_{*}[\Gamma]\right)-\sum_{i=1}^{r} \mathcal{F}\left(j_{*}\left([C]-\frac{1}{2} K-[\Gamma]\right)\right) \cdot \frac{(-h)^{i}}{i !},
\end{aligned}
$$

where we identify $\mathrm{CH}(\mathbb{P} \times J)=\mathrm{CH}(J)[h] /\left(h^{r+1}\right)$, with $h$ the hyperplane class on $\mathbb{P}$.

Proof. Consider the rank $r$ vector bundle $E$ on $C$ whose fibre at a point $P$ is $H^{0}(C, \Gamma-P)$. More formally, let $\pi_{1}, \pi_{2}: C \times C \rightarrow C$ be the projections; then $E:=\pi_{2, *}\left(\pi_{1}^{*} O_{C}(\Gamma) \otimes O_{C \times C}(-\Delta)\right)$, which by our assumption that $|\Gamma|$ is base-point free is indeed locally free of rank $r$, and which sits in an exact sequence

$$
0 \longrightarrow E \longrightarrow V \otimes_{k} O_{C} \longrightarrow O_{C}(\Gamma) \longrightarrow 0 \text {. }
$$

As $Y \rightarrow C$ is the projective bundle associated to $E$ we have

$$
\mathrm{CH}(Y)=\mathrm{CH}(C)[H] /\left(H^{r}-[\Gamma] \cdot H^{r-1}\right),
$$

where $H:=c_{1}\left(O_{Y}(1)\right)$. If we have classes $\alpha_{j} \in \mathrm{CH}(C)$, almost all zero, then $q_{1, *}\left(\sum_{j \geq 0} \alpha_{j} H^{j}\right)=\alpha_{r-1}+[\Gamma] \cdot \alpha_{r}$. Also note that $O_{Y}(1)=q_{2}^{*} O_{\mathbb{P}}(1)$, so if $h:=c_{1}\left(O_{\mathbb{P}}(1)\right) \in \mathrm{CH}^{1}(\mathbb{P})$ is the hyperplane class on $\mathbb{P}$, we have $H=q_{2}^{*}(h)$.

Let $p_{1}: Y \times J \rightarrow Y$ and $p_{2}: Y \times J \rightarrow J$ be the projections. GRR gives

$$
\begin{aligned}
\operatorname{ch}\left(\left(q_{2} \times \mathrm{id}\right)_{*} \mathcal{M}\right) & =\left(q_{2} \times \mathrm{id}\right)_{*}\left(\operatorname{ch}(\mathcal{M}) \cdot p_{1}^{*} \operatorname{Td}(Y / \mathbb{P})\right) \\
& =\sum_{i=0}^{r} p_{2, *}\left(\operatorname{ch}(\mathcal{M}) \cdot p_{1}^{*}\left(\operatorname{Td}(Y / \mathbb{P}) \cdot H^{r-i}\right)\right) \cdot h^{i}
\end{aligned}
$$

We calculate $p_{2, *}$ by first pushing down to $C \times J$ and then pushing down via the projection map $\mathrm{pr}_{2}: C \times J \rightarrow J$. This gives

$$
\begin{aligned}
\operatorname{ch}\left(\left(q_{2} \times \mathrm{id}\right)_{*} \mathcal{M}\right) & =\sum_{i=0}^{r} \operatorname{pr}_{2, *}\left(\operatorname{ch}(\mathscr{L}) \cdot \operatorname{pr}_{1}^{*} q_{1, *}\left(\operatorname{Td}(Y / \mathbb{P}) \cdot H^{r-i}\right)\right) \cdot h^{i} \\
& =\sum_{i=0}^{r} \mathcal{F}\left(j_{*} q_{1, *}\left(\operatorname{Td}(Y / \mathbb{P}) \cdot H^{r-i}\right)\right) \cdot h^{i}
\end{aligned}
$$


Next we use that $\operatorname{Td}(Y / \mathbb{P})=\operatorname{Td}(Y / C) \cdot q_{1}^{*} \operatorname{Td}(C) \cdot q_{2}^{*} \operatorname{Td}(\mathbb{P})^{-1}$. But $q_{2}^{*} \operatorname{Td}(\mathbb{P})=$ $\operatorname{Td}\left(V \otimes_{k} O_{Y}(1)\right)$, so using the exact sequences (12) and

$$
0 \longrightarrow O_{Y} \longrightarrow\left(q_{1}^{*} E\right)(1) \longrightarrow T_{Y / C} \longrightarrow 0
$$

we find that $\operatorname{Td}(Y / \mathbb{P})=q_{1}^{*} \operatorname{Td}(C) \cdot \operatorname{Td}\left(q_{1}^{*} O_{C}(\Gamma) \otimes O_{Y}(1)\right)^{-1}$. Now

$$
\begin{aligned}
\operatorname{Td}\left(q_{1}^{*} O_{C}(\Gamma) \otimes O_{Y}(1)\right)^{-1} & =\sum_{m \geq 0} \frac{(-1)^{m}}{(m+1) !} \cdot([\Gamma]+H)^{m} \\
& =\sum_{m \geq 0} \frac{(-1)^{m}}{(m+1) !} \cdot H^{m}+[\Gamma] \cdot \sum_{m \geq 1} \frac{(-1)^{m} \cdot m}{(m+1) !} \cdot H^{m-1},
\end{aligned}
$$

so we find

$$
q_{1, *}\left(\operatorname{Td}(Y / \mathbb{P}) \cdot H^{r-i}\right)= \begin{cases}{[\Gamma]} & \text { if } i=0 \\ \frac{(-1)^{i-1}}{i !} \cdot\left([C]-\frac{1}{2} K\right) \cdot([C]-[\Gamma]) & \text { if } i>0\end{cases}
$$

Putting this back into (13) we get the proposition.

4.3. To apply Proposition 4.2 we need some notation. We introduce classes $c_{i}(\Gamma)$ that are the Chern classes of a vector bundle for which the classes $a_{n}(\Gamma)$ defined in 3.4 are the components of the Chern character. Concretely, choose a divisor $\Gamma$ in the linear system such that $\Gamma=Q_{1}+Q_{2}+\cdots+Q_{d}$ for distinct points $Q_{i} \in C$. Let $L_{i}$ be the restriction of $\mathscr{L}$ to $\left\{Q_{i}\right\} \times J$, and define $W:=L_{1} \oplus \cdots \oplus L_{d}$. We then have $a_{m}(\Gamma)=\operatorname{ch}_{m}(W)$. Now define

$$
c_{i}(\Gamma):=c_{i}(W) \quad \text { and } \quad c_{t}(\Gamma):=c_{t}(W) .
$$

By construction we have

$$
\exp \left(\sum_{m \geq 1}(-1)^{m-1}(m-1) ! a_{m}(\Gamma) t^{m}\right)=c_{t}(\Gamma)
$$

4.4. Consider the situation as in 4.1. Let $\mathcal{N}$ be the vector bundle of rank $d$ on $\mathbb{P} \times J$ given by $\mathcal{N}:=\left(q_{2} \times \mathrm{id}\right)_{*} \mathcal{M} \otimes O_{\mathbb{P}}(1)$. Our GRR calculation gives

$$
\operatorname{ch}(\mathcal{N})=\mathcal{F}\left(j_{*}[\Gamma]\right)+\sum_{n \geq 1} \mathcal{F}\left(j_{*}\left([C]-\frac{1}{2} K\right)\right) \cdot \frac{h^{n}}{n !},
$$

so

$$
\operatorname{ch}_{0}(\mathcal{N})=a_{0}(\Gamma)=d
$$


and

$$
\operatorname{ch}_{m}(\mathcal{N})=a_{m}(\Gamma)+\sum_{n=1}^{m} \frac{p_{m-n}-\frac{1}{2} a_{m-n}(K)}{n !} h^{n} \quad \text { for } m \geq 1 .
$$

The non-trivial information we have is that

$$
c_{t}(\mathcal{N})=\exp \left(\sum_{m \geq 1}(-1)^{m-1}(m-1) ! \operatorname{ch}_{m}(\mathcal{N}) t^{m}\right)
$$

is a polynomial in $t$ of degree $\leq d$. The RHS equals

$$
\begin{aligned}
& c_{t}(\Gamma) \cdot \exp \left(\sum_{1 \leq n<m} \frac{(-1)^{m-1}(m-1) ! p_{m-n} h^{n} t^{m}}{n !}\right) \\
& \cdot \exp \left(\sum_{1 \leq n \leq m} \frac{(-1)^{m-1}(m-1) ! a_{m-n}(K) h^{n} t^{m}}{2 \cdot n !}\right) .
\end{aligned}
$$

(Note that we may take $n<m$ in the second factor, as $p_{0}=0$.) Separating terms according to their type in the Beauville decomposition this gives that for every $j \geq 0$ the expression

$$
\begin{aligned}
& c_{t}(\Gamma) \cdot\left(\sum_{1 \leq n<m} \frac{(-1)^{m-1}(m-1) ! p_{m-n} h^{n} t^{m}}{n !}\right)^{j} \\
& \cdot \exp \left(\sum_{1 \leq n \leq m} \frac{(-1)^{m-1}(m-1) ! a_{m-n}(K) h^{n} t^{m}}{2 \cdot n !}\right)
\end{aligned}
$$

is a polynomial in $t$ of degree at most $d$.

4.5. In the relation we have obtained we take $j=r$. Note that $h^{r+1}=0$. Hence

$$
\begin{aligned}
\left(\sum_{1 \leq n<m} \frac{(-1)^{m-1}(m-1) ! p_{m-n} h^{n} t^{m}}{n !}\right)^{r} & =\left(\sum_{m \geq 2}(-1)^{m-1}(m-1) ! p_{m-1} h t^{m}\right)^{r} \\
& =\left(\sum_{m \geq 1}(-1)^{m} m ! p_{m} t^{m}\right)^{r} \cdot h^{r} t^{r}
\end{aligned}
$$

We define $B(i, s)$ as $(-1)^{i}$ times the coefficient of $t^{i}$ in $\left(\sum_{m \geq 1}(-1)^{m} m ! p_{m} t^{m}\right)^{s}$; so

$$
B(i, s)=\sum_{\substack{m_{1}, \ldots, m_{s} \\ m_{1}+\cdots+m_{s}=i}} m_{1} ! \ldots m_{s} ! \cdot p_{m_{1}} \ldots p_{m_{s}} .
$$

Then the relation we find is that $c_{t}(\Gamma) \cdot \sum_{i \geq 1}(-1)^{i} B(i, r) t^{i}$ is a polynomial in $t$ of degree at most $d-r$. In particular, this gives the following result. 
4.6 Theorem. Let $C$ be a curve with a $g_{d}^{r}$. Let $\Gamma$ be a divisor in the linear system. Define elements $c_{n}(\Gamma)$ as in 4.3 and elements $B(i, s)$ as in (15). Then we have

$B(i, r)=c_{1}(\Gamma) \cdot B(i-1, r)-c_{2}(\Gamma) \cdot B(i-2, r)+\cdots+(-1)^{i} c_{i-1}(\Gamma) \cdot B(1, r)$.

for all $i>d-r$.

Note that without loss of generality we may assume that the $g_{d}^{r}$ is complete and base-point free. Passing to the quotient of $\mathrm{CH}(J)$ modulo algebraic equivalence we recover Theorem 2.14.

4.7. The relations in Theorem 4.6 are only the tip of the iceberg. There is an abundance of further relations. There are at least three methods we can use: (i) Take other values for $j$ in (14); (ii) Apply the operator $\mathscr{D}$ to relations we have found; (iii) Use that if the curve has a $g_{d}^{r}$ then it also has a $g_{d-1}^{r-1}$. The main difficulty is to extract manageable information, and here we have little to offer. So we confine ourselves to some simple examples of how we can get more relations.

4.8 Example. We consider the relations we obtain by taking $j=r-1$ in (14). We have

$$
\left(\sum_{1 \leq n<m} \frac{(-1)^{m-1}(m-1) ! p_{m-n} h^{n} t^{m}}{n !}\right)^{r-1}=A_{1} \cdot h^{r-1} t^{r-1}+A_{2} \cdot h^{r} t^{r},
$$

with

$$
A_{1}=\left(\sum_{m \geq 1}(-1)^{m} m ! p_{m} t^{m}\right)^{r-1}=\sum_{v>0}(-1)^{v} B(v, r-1) t^{\nu}
$$

and

$$
\begin{aligned}
A_{2} & =\frac{r-1}{2} \cdot\left(\sum_{m \geq 1}(-1)^{m+1}(m+1) ! p_{m} t^{m}\right) \cdot\left(\sum_{m \geq 1}(-1)^{m} m ! p_{m} t^{m}\right)^{r-2} \\
& =\frac{r-1}{2} \cdot\left(\sum_{m \geq 1}(-1)^{m+1}(m+1) ! p_{m} t^{m}\right) \cdot\left(\sum_{\nu>0}(-1)^{v} B(v, r-2) t^{v}\right) .
\end{aligned}
$$

On the other hand,

$$
\begin{aligned}
& \exp \left(\sum_{1 \leq n \leq m} \frac{(-1)^{m-1}(m-1) ! a_{m-n}(K) h^{n} t^{m}}{2 \cdot n !}\right) \\
& \quad=1+\frac{1}{2} \cdot\left(\sum_{m \geq 0}(-1)^{m} m ! a_{m}(K) t^{m}\right) \cdot h t \quad\left(\bmod h^{2}\right),
\end{aligned}
$$


So we obtain from 4.4 that $c_{t}(\Gamma) \cdot \sum_{v>0}(-1)^{v} B(v, r-1) t^{v}$ is a polynomial of degree $\leq d+1-r$, and that

$$
\begin{aligned}
c_{t}(\Gamma) & \cdot\left\{\left(\sum_{\nu>0}(-1)^{v} B(\nu, r-1) t^{\nu}\right) \cdot\left(\sum_{m \geq 0}(-1)^{m} m ! a_{m}(K) t^{m}\right)\right. \\
& \left.+(r-1) \cdot\left(\sum_{m \geq 1}(-1)^{m+1}(m+1) ! p_{m} t^{m}\right) \cdot\left(\sum_{\nu>0}(-1)^{v} B(\nu, r-2) t^{\nu}\right)\right\}
\end{aligned}
$$

is a polynomial of degree $\leq d-r$.

Concretely this means that

$$
\sum_{n=0}^{i-1}(-1)^{n} c_{n}(\Gamma) B(i-n, r-1)=0 \quad \text { for all } i \geq d+2-r
$$

and

$$
\begin{aligned}
& \sum_{n=0}^{i} c_{n}(\Gamma) \cdot\left\{\sum_{\nu>0}(-1)^{n-i}(n-i-v) ! B(\nu, r-1) a_{n-i-v}(K)\right. \\
& \left.\quad+(r-1) \cdot \sum_{\nu>0}(-1)^{n-i+1}(n-i+1-v) ! B(\nu, r-2) p_{n-i-v}\right\}=0
\end{aligned}
$$

for all $i \geq d+1-r$.

4.9 Example. Consider a curve with a $g_{d}^{1}$; so we take $r=1$ in the above. Theorem 4.6 gives

$$
p_{i}=\frac{1}{i !} \cdot \sum_{\nu=1}^{i-1}(-1)^{i+v+1} \nu ! c_{i-v}(\Gamma) p_{v} \quad \text { for all } i \geq d .
$$

Applying $\mathscr{D}$ we get, using Theorem 3.6,

$$
\begin{aligned}
-q_{i-1}= & \sum_{\nu=1}^{i-1} \frac{(-1)^{i+v} v !}{i !} c_{i-v}(\Gamma) q_{\nu-1} \\
& +\sum_{\nu=1}^{i-1} \sum_{m \geq 1} \frac{(-1)^{i+v+1} v !}{i !}\left(\begin{array}{c}
m+v-1 \\
v
\end{array}\right) a_{m+v-1}(\Gamma) \partial_{a_{m}(\Gamma)}\left(c_{i-v}(\Gamma)\right) .
\end{aligned}
$$

Now use that $\partial_{a_{m}(\Gamma)}\left(c_{k}(\Gamma)\right)=(-1)^{m-1}(m-1) ! c_{k-m}(\Gamma)$. So we find that

$$
\begin{aligned}
-q_{i-1}= & \sum_{\nu=1}^{i-1} \frac{(-1)^{i+v} v !}{i !} c_{i-v}(\Gamma) q_{v-1} \\
& +\sum_{\nu \geq 1} \frac{(-1)^{i+1+v}(\nu-1) \cdot v !}{i !} \cdot a_{v}(\Gamma) c_{i-1-v}(\Gamma)
\end{aligned}
$$

for all $i \geq d$. 
4.10. Next consider a curve with a $g_{d}^{2}$, i.e., we take $r=2$. The first type of relation we have is the one give by Theorem 4.6, which for every $i \geq d-1$ gives an expression of $B(i, 2)=\sum_{m=1}^{i-1} m !(i-m) ! p_{m} p_{i-m}$ as a linear combination of terms $c_{n}(\Gamma) \cdot B(i-n, 2)$.

Next we can use that the $g_{d}^{2}$ gives rise, in several ways, to a $g_{d-1}^{1}$. Concretely, let $\Gamma=Q_{1}+\cdots+Q_{d}$ be one of the divisors of the $g_{d}^{2}$, where the $Q_{i}$ are distinct. For $s \in\{1, \ldots, d\}$ let $\Gamma^{(s)}:=\Gamma-Q_{s}$. Applying Theorem 4.6 to the $g_{d-1}^{1}$ 's thus obtained we get relations

$$
p_{i}=\frac{1}{i !} \cdot \sum_{\nu=1}^{i-1}(-1)^{i+v+1} \nu ! c_{i-v}\left(\Gamma^{(s)}\right) p_{v}
$$

for all $i \geq d-1$ and all $s \in\{1, \ldots, d\}$. Summing over $s$ this gives

$$
p_{i}=\frac{1}{d \cdot i !} \cdot \sum_{\nu=1}^{i-1}(-1)^{i+v+1} \nu !(d+v-i) c_{i-v}(\Gamma) p_{\nu} \quad \text { for all } i \geq d-1 .
$$

Thirdly we can apply what we found in 4.8. Equation (16) gives

$$
p_{i}=\frac{1}{i !} \cdot \sum_{\nu=1}^{i-1}(-1)^{i+v+1} v ! c_{i-v}(\Gamma) p_{v} \quad \text { for all } i \geq d
$$

which also follows from (18). Equation (17) gives

$$
\sum_{n=0}^{i} \sum_{\nu=1}^{n-i}(-1)^{n-i}(n-i-v) ! \nu ! p_{\nu} c_{n}(\Gamma) a_{n-i-v}(K)=0 .
$$

(Recall that the elements $a_{l}(K)$ are essentially the classes $q_{l}$; see (4).)

Finally we can apply the operator $\mathscr{D}$ to relations that we have found. For instance, applying $\mathscr{D}$ to the relation in Theorem 4.6 we find a relation

$$
\begin{aligned}
0=\sum_{m=1}^{i-1}(-1)^{m+1} m \cdot(m+1) ! c_{i-m-1}(\Gamma) p_{m} \\
\quad+2 \cdot \sum_{m=1}^{i-1} \sum_{u=0}^{i-m-1}(-1)^{m+u} u \cdot u ! m ! a_{u}(\Gamma) c_{i-m-u-1}(\Gamma) p_{m} \\
\quad+2 \cdot \sum_{m=1}^{i-1} \sum_{u=0}^{i-m-1}(-1)^{m+u}(u+1) ! m ! q_{u} c_{i-m-u-1}(\Gamma) p_{m}=0
\end{aligned}
$$

for all $i \geq d-1$. Using this identity we can express the classes $p_{i}$ for $i \geq d-2$ as linear combinations of the $p_{i-v}(v>0)$ with coefficients in $\mathbb{Q}\left[q_{m}, a_{m}(\Gamma) ; m \geq 1\right]$. It 
seems that these relations (for $i \geq d-1$ ) do not, in general, follow from the relations we have already obtained in (18), (19) and (20).

In conclusion, we have relations between the generators $p_{m}, q_{m}$ and $a_{m}(\Gamma)$ galore. At this stage, however, we do not have a simple set of generators for the whole ideal of relations.

4.11. A general curve of genus $g$ has gonality $\left\lceil\frac{g}{2}\right\rceil+1$. As Polishchuk shows in [16], Proposition 4.2, in the small tautological ring taut $(C)$ all classes $p_{m}$ with $m \geq(g / 2)+1$ lie in the ideal generated by the classes $q_{n}$ for $n \geq 1$. So one might expect that if the curve has a $g_{d}^{1}$, all classes $p_{n}$ with $n \geq d$ should lie in the ideal $\left(\left\{q_{n}\right\}_{n \geq 1}\right)$, at least for a suitable choice of a base point. This, however, is probably too optimistic, except in special situations. (The main difficulty in disproving this lies in the dependence on the base point.)

As an example of a special situation, suppose the curve has a $g_{d}^{r}$ of divisors $\Gamma$ that are (rational) multiples of the canonical class $K$. In this case all $c_{i}(\Gamma)$ are in the ring $\mathbb{Q}\left[q_{l} ; l \geq 1\right]$. We find, for all $i>d-r$, that $B(i, r)$ is an element of the small tautological ring, and lies in the ideal generated by the classes $q_{l}$. We can push this a bit further by applying the operator $\mathscr{D}$. Before we state the result let us formulate a lemma.

4.12 Lemma. Given integers $i>s$, define

$$
C(i, s):=\sum_{\substack{m_{1}, \ldots, m_{s} \geq 2 \\ m_{1}+\cdots+m_{s}=i}} m_{1} ! \ldots m_{s} ! \cdot p_{m_{1}} \ldots p_{m_{s}} .
$$

Then

$$
\mathscr{D}^{s-1}(C(i, s))=\frac{i !(i-s-1) !}{(i-2 s) !} \cdot p_{i+1-s}
$$

for all $i \geq 0$ and $s \geq 1$.

Proof. Change to the variables $y_{n}:=(n+1) ! p_{n+1}$. Then $\mathscr{D}$ acts as the operator $\varepsilon_{1}+\varepsilon_{2}$ with

$$
\mathcal{E}_{1}:=\sum_{m, n \geq 1} y_{m+n} \partial_{m} \partial_{n} \quad \text { and } \quad \mathcal{E}_{2}:=\sum_{m, n \geq 1} \frac{m+n}{2} \cdot y_{m+n} \partial_{m} \partial_{n} .
$$

Letting

$$
C^{\prime}(k, s):=\sum_{\substack{n_{1}, \ldots, n_{s} \\ n_{1}+\cdots+n_{s}=k}} y_{n_{1}} \ldots y_{n_{s}}
$$

it is clear that $D^{s-1}\left(C^{\prime}(k, s)\right)=c \cdot y_{k}$ for some rational number $c$. Our goal is to prove that $c=(k+s) !(k-1) ! /(k-s) !(k+1)$ !. This can be checked by evaluation at the vector $1:=(1,1, \ldots)$. 
On $\mathbb{Q}\left[y_{1}, y_{2}, \ldots\right]$ consider gradings $d$ and $\delta$ given by $d\left(y_{i}\right)=1$ and $\delta\left(y_{i}\right)=i$. Suppose $f$ is homogeneous for both. Then one finds without difficulty (reduce to monomials) that $\varepsilon_{1}(f)(\mathbf{1})=d(d-1) \cdot f(\mathbf{1})$ and $\varepsilon_{2}(f)(\mathbf{1})=\delta(d-1) \cdot f(\mathbf{1})$. Note further that $\mathcal{E}_{1}$ and $\mathcal{E}_{2}$ both decrease $d$ by 1 and preserve $\delta$. Hence, by induction on $n \leq s-1$,

$$
\mathscr{D}^{n}\left(C^{\prime}(k, s)\right)(\mathbf{1})=\frac{(k-1) !(k+s) !}{(k-s) !(k+s-n) !(s-1-n) !},
$$

and taking $n=s-1$ gives the result.

4.13 Proposition. Assume the curve $C$ has a $g_{d}^{r}$ of divisors $\Gamma$ such that $\Gamma \in \mathbb{Q} \cdot K$ in $\mathrm{CH}^{1}(C)$. Given integers $g, i$ and $r$, let

$$
\Psi(g, i, r):=\sum_{a=0}^{r-1}\left(\begin{array}{c}
i-g \\
a
\end{array}\right)\left(\begin{array}{l}
i-a \\
r-a
\end{array}\right)\left(\begin{array}{l}
i-r-1 \\
r-1-a
\end{array}\right)
$$

Then $p_{k}$ lies in the ideal generated by the classes $q_{l}$, for all $k>d+1-2 r$ with $\Psi(g, k+r-1, r) \neq 0$.

A similar result has been obtained independently by $\mathrm{Fu}$ and Herbaut in [8]. Their result assumes the nonvanishing of a certain numerical factor $A(r, d, g)$, called the Castelnuovo number. We have the relation $\Psi(g, d-r+1, r)=(d-2 r+2)$. $A(r, d, g)$.

Proof. The idea is to apply $\mathscr{D}^{r-1}$ to the relation $B(i, r)=\sum_{n=1}^{i-1}(-1)^{n-1} c_{n}(\Gamma) B(i-$ $n, r)$ for $i>d-r$ that we have obtained in Theorem 4.6. Since all elements $c_{n}(\Gamma)$ are in the ring $\mathbb{Q}\left[q_{l} ; l \geq 1\right]$ it is easy to see that $\mathscr{D}^{r-1}$ applied to the RHS gives an element in the small tautological ring that lies in the ideal generated by the classes $q_{l}$. (Keep track of the number of terms $p$ and $q$ after application of the operator $\mathscr{D}$, as in the proof of [16], Proposition 4.2.)

On the other hand, it is clear that $\mathscr{D}^{r-1}(B(i, r))$ is a multiple of $p_{i+1-r}$, and in fact we claim that

$$
D^{r-1}(B(i, r))=r !(r-1) !(i-r) ! \cdot \Psi(g, i, r) \cdot p_{i+1-r} .
$$

This indeed gives the stated result. To prove this identity, note that $B(i, s)=$ $\sum_{a=0}^{s}\left(\begin{array}{l}s \\ a\end{array}\right) p_{1}^{a} C(i-a, s-a)$. (To see this use that $B(i, s)$ is the coefficient of $t^{i}$ in $\left(\sum_{m \geq 1} m ! p_{m} t^{m}\right)^{s}$, and separate the terms with $m=1$ from those with $m \geq 2$.) Now we use Lemma 2.4, which (after replacing $x_{1}$ by $p_{1}$ ) is still valid in the present situation. We find

$$
\mathscr{D}^{r-1}(B(i, r))=\sum_{a=0}^{r}\left(\begin{array}{l}
r \\
a
\end{array}\right) \mathscr{D}^{r-1}\left(p_{1}^{a} C(i-a, r-a)\right)=
$$




$$
\begin{gathered}
=\sum_{a=0}^{r} \sum_{b=0}^{\min (a, r-1)}\left(\begin{array}{l}
r \\
a
\end{array}\right) \frac{(r-1) !}{(r-1-b) !} \frac{a !}{(a-b) !}\left(\begin{array}{c}
i-a-g+b \\
b
\end{array}\right) \\
\cdot p_{1}^{a-b}{D^{r-1-b}}^{r-b(i-a, r-a)) .}
\end{gathered}
$$

But it is clear from the explicit shape of the operator $\mathscr{D}_{\text {that }} \mathscr{D}^{r-1-b}(C(i-a, r-a))=$ 0 if $b<a$. So we find, using Lemma 4.12,

$$
\begin{aligned}
\mathscr{D}^{r-1}(B(i, r)) & =\sum_{a=0}^{r-1}\left(\begin{array}{l}
r \\
a
\end{array}\right) \frac{(r-1) ! a !}{(r-1-a) !}\left(\begin{array}{c}
i-g \\
a
\end{array}\right) \frac{(i-a) !(i-r-1) !}{(i-2 r+a) !} \cdot p_{i+1-r} \\
& =r !(r-1) !(i-r) ! \cdot \Psi(g, i, r) \cdot p_{i+1-r}
\end{aligned}
$$

Setting $k=i+1-r$ we obtain the proposition.

Of course, if $C$ has a $g_{d}^{r}$ then one can also proceed as in Remark 2.15 and use what we have found in Example 4.9. This gives expressions for the classes $p_{k}$ for $k>d+2-2 r$, or even $k \geq d+1-2 r$, as linear combinations of elements $c_{k-v}\left(\Gamma^{\prime}\right) p_{v}$ for $v<k$, where $\left|\Gamma^{\prime}\right|$ is some other linear system on $C$ that calculates the Clifford index.

\section{References}

[1] A. Beauville, Quelques remarques sur la transformation de Fourier dans l'anneau de Chow d'une variété abélienne. In Algebraic geometry (Tokyo/Kyoto, 1982), M. Raynaud and T. Shioda, eds., Lecture Notes in Math. 1016, Springer, Berlin 1983, 238-260. Zbl 0526.14001 MR 0726428

[2] A. Beauville, Algebraic cycles on Jacobian varieties. Compositio Math. 140 (2004), 683-688. Zbl 1062.14011 MR 2041776

[3] G. Ceresa, $C$ is not algebraically equivalent to $C^{-}$in its Jacobian. Ann. of Math. 117 (1983), 285-291. Zbl 0538.14024 MR 0690847

[4] E. Colombo and B. van Geemen, Note on curves in a Jacobian. Compositio Math. 88 (1993), 333-353. Zbl 0802.14002 MR 1241954

[5] M. Coppens and G. Martens, Secant spaces and Clifford's theorem. Compositio Math. 78 (1991), 193-212. Zbl 0741.14035 MR 1104787

[6] D. Eisenbud, H. Lange, G. Martens and F.-O. Schreyer, The Clifford dimension of a projective curve. Compositio Math. 72 (1989), 173-204. Zbl 0703.14020 MR 1030141

[7] N. Fakhruddin, Algebraic cycles on generic abelian varieties. Compositio Math. 100(1996), 101-119. Zbl 0871.14005 MR 1377410

[8] B. Fu and F. Herbaut, On the tautological ring of a Jacobian modulo rational equivalence. Geom. Dedicata 129 (2007), 145-153. Zbl 05177027 MR 2353988 
[9] G. van der Geer and A. Kouvidakis, Cycle relations on Jacobian varieties. Compositio Math. 143 (2007), 900-908. Zbl 1125.14005 MR 2339832

[10] F. Herbaut, Algebraic cycles on the Jacobian of a curve with a linear system of given dimension. Compositio Math. 143 (2007), 883-899. Zbl 05177027 MR 2339831

[11] A. Ikeda, Algebraic cycles and infinitesimal invariants on Jacobian varieties. J. Algebraic Geom. 12 (2003), 573-603. Zbl 1077.14508 MR 1966027

[12] U. Jannsen, Motivic sheaves and filtrations on Chow groups. In Motives (Seattle, WA, 1991), U. Jannsen, S. Kleiman and J-P. Serre, eds., Proc. Symp. Pure Math. 55, Amer. Math. Soc., Providence, RI, 1994, 245-302. Zbl 0811.14004 MR 1265533

[13] K. Künnemann, A Lefschetz decomposition for Chow motives of abelian schemes. Invent. Math. 113 (1993), 85-102. Zbl 0806.14001 MR 1223225

[14] B. Moonen and A. Polishchuk, Algebraic cycles on the relative symmetric powers and on the relative Jacobian of a family of curves. II. Preprint, 2008. arXiv:0805.3621v1

[15] A. Polishchuk, Universal algebraic equivalences between tautological cycles on Jacobians of curves. Math. Z. 251 (2005), 875-897. Zbl 1083.14030 MR 2190148

[16] A. Polishchuk, Lie symmetries of the Chow group of a Jacobian and the tautological subring. J. Alg. Geom. 16 (2007), 459-476. Zbl 1123.14002 MR 2306276

[17] A. Polishchuk, Algebraic cycles on the relative symmetric powers and on the relative Jacobian of a family of curves. I. Selecta Math. (N.S.) 13 (2007), 531-569 Zbl 1143.14009 MR 2383605

[18] A. Polishchuk, Fourier-stable subrings in the Chow rings of abelian varieties. Math. Res. Lett. 15 (2008), 705-714. Zbl 05492435 MR 2424907

Received July 9, 2007

Ben Moonen, University of Amsterdam, KdV Institute for Mathematics, PO Box 94248, 1090 GE Amsterdam, The Netherlands

E-mail: b.j.j.moonen@uva.nl 